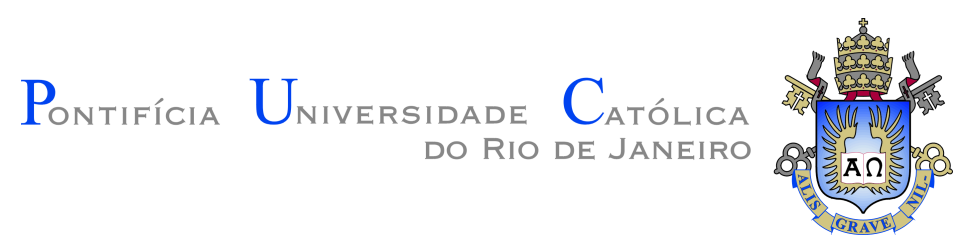

Marina Dietze Monteiro

\title{
A Novel Semiparametric Structural Model for Electricity Forward Curves
}

Dissertação de Mestrado

Dissertation presented to the Programa de Pós-graduação em Engenharia Elétrica of PUC-Rio in partial fulfillment of the requirements for the degree of Mestre em Engenharia Elétrica.

Advisor : Prof. Alexandre Street de Aguiar

Co-advisor: $\quad$ Prof. Davi Michel Valladão 


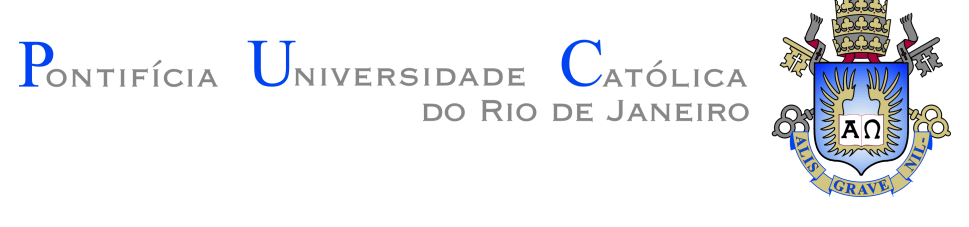

Marina Dietze Monteiro

\section{A Novel Semiparametric Structural Model for Electricity Forward Curves}

Dissertation presented to the Programa de Pós-graduação em Engenharia Elétrica of PUC-Rio in partial fulfillment of the requirements for the degree of Mestre em Engenharia Elétrica. Approved by the Examination Committee:

Prof. Davi Michel Valladão

Co-advisor

Departamento de Engenharia Industrial - PUC-Rio

Prof. Cristiano Augusto Coelho Fernandes

Departamento de Engenharia Elétrica - PUC-Rio

Prof. Stein-Erik Fleten

Norwegian University of Science and Technology, NTNU

Rio de Janeiro, September the 30th, 2020 
All rights reserved.

\section{Marina Dietze Monteiro}

Marina Dietze Monteiro received her B.Sc. degree in Electrical Engineering in 2018 from the Universidade Federal Fluminense (UFF), Brazil. During her undergraduate program, she participated in the Research for Undergraduates program (Summer 2017) at Texas Tech University. Since 2019, she has been part of the Laboratory of Applied Mathematical Programming and Statistics (LAMPS) in the Department of Electrical Engineering at the Pontifícia Universidade Católica do Rio de Janeiro (PUC-RIO).

Bibliographic data

Monteiro, Marina Dietze

A novel semiparametric structural model for electricity forward curves / Marina Dietze Monteiro; advisor: Alexandre Street de Aguiar; co-advisor: Davi Michel Valladão. - 2020.

$74 \mathrm{f}$ : il. color. ; $30 \mathrm{~cm}$

Dissertação (mestrado) - Pontifícia Universidade Católica do Rio de Janeiro, Departamento de , 2020.

Inclui bibliografia

1. Engenharia Elétrica - Teses. 2. Curvas forward de eletricidade. 3. Suavidade. 4. Análise de componentes principais. I. Street, Alexandre. II. Valladão, Davi Michel. III. Pontifícia Universidade Católica do Rio de Janeiro. Departamento de . IV. Título. 


\section{Acknowledgments}

First, I would like to thank my parents, Jorge and Carla, and my sister, Julia, to the unlimited support and love throughout my entire life.

To my advisor, Alexandre Street, who gave me the opportunity to join the Master's program at PUC-Rio and be part of the Lamps team. Thank you for always supporting me and for the high-quality conversations.

To my co-advisor, Davi Valladão, that assisted me closely during this study. I'm grateful for all the support and numerous and essential discussions in this dissertation's development.

I would like to thank my boyfriend, Eduardo, to always believing in me when I needed the most and to our valuable partnership.

To the unconditional friendship and support from my dearest friend Mariana.

To my friend Luíza, who begun this journey with me and brought even more value to these two years.

To all my friends at Lamps, thank you for the warm and learning environment.

A special thanks to the team: Carol, Iago, Guilherme, Luíza, Bruno, Cristiano, Pedro and Stein-Erik. This work was a result of fantastic discussions of an extremely qualified group.

This study was financed in part by the Coordenação de Aperfeiçoamento de Pessoal de Nível Superior - Brasil (CAPES) - Finance Code 001.

This study was financed by the Fundação de Amparo à Pesquisa do Estado do Rio de Janeiro (FAPERJ) and Eneva. 


\section{Abstract}

Dietze Monteiro, Marina; Street, Alexandre (Advisor); Valladão, Davi Michel (Co-Advisor). A Novel Semiparametric Structural Model for Electricity Forward Curves. Rio de Janeiro, 2020. 74p. Dissertação de Mestrado - Departamento de , Pontifícia Universidade Católica do Rio de Janeiro.

Hedging against spot price volatilities becomes increasingly important in deregulated power markets. Therefore, being able to model electricity forward prices is crucial in a competitive environment. Electricity differs from other commodities due to its limited storability and transportability. Furthermore, its derivatives are associated with a delivery period during which electricity is continuously delivered, implying on referring to power forwards as swaps. These peculiarities make the modeling of electricity contract prices a non-trivial task, where traditional models must be adapted to address the mentioned characteristics. In this context, we propose a novel semiparametric structural model to compute a continuous daily forward curve of electricity through maximum smoothness criterion. In addition, elementary forward contracts can be represented by any parametric structure for seasonality or even for exogenous variables. Our framework acknowledges the overlapped swaps and allows an analysis of arbitrage opportunities observed in power markets. The smooth forward curve is computed by a hierarchical optimization problem able to handle scarce data sets from low-liquidity markets. PCA results corroborate our framework's capability to explain a high percentage of variance with only a few factors.

\section{Keywords}

Electricity forward curves; Smoothing; Principal component analysis. 


\section{Resumo}

Dietze Monteiro, Marina; Street, Alexandre; Valladão, Davi Michel. Modelo Estrutural Semi-Paramétrico para Curvas Forward de Eletricidade. Rio de Janeiro, 2020. 74p. Dissertação de Mestrado - Departamento de , Pontifícia Universidade Católica do Rio de Janeiro.

A proteção contra a volatilidade dos preços spot torna-se cada vez mais importante nos mercados de energia desverticalizados. Portanto, ser capaz de modelar preços forward e futuros de eletricidade é crucial em um ambiente competitivo. A eletricidade difere de outras commodities devido à sua capacidade de armazenamento e transporte limitados. Além disso, seus derivativos estão associados a um período de entrega durante o qual a energia é concedida continuamente, o que implica em muitas vezes os contratos de eletricidades serem denominados swaps. Tais peculiaridades tornam a modelagem de preços de contratos de energia elétrica uma tarefa não trivial, onde os modelos tradicionais devem ser adaptados para atender às características mencionadas. Neste contexto, foi proposto um modelo estrutural semi-paramétrico para obtenção de uma curva forward de eletricidade contínua e diária através de critérios de máxima suavidade. Ademais, os contratos forward elementares podem ser representados por qualquer estrutura paramétrica para sazonalidade ou mesmo para variáveis exógenas. Nossa estrutura reconhece a sobreposição dos swaps e permite uma análise das oportunidades de arbitragem observadas nos mercados de energia. A curva forward é calculada por um problema de otimização hierárquico capaz de lidar com conjuntos de dados escassos de mercados com baixa liquidez. Os resultados do PCA corroboram a capacidade do modelo em explicar uma alta porcentagem da variância com apenas alguns fatores.

\section{Palavras-chave}

Curvas forward de eletricidade; Suavidade; Análise de componentes principais. 


\section{Table of contents}

1 Introduction $\quad 11$

$\begin{array}{lll}1.1 & \text { Objective and Contributions } & 15\end{array}$

2 Literature Review $\quad 16$

$\begin{array}{lll}2.1 & \text { Spot Modeling } & 16\end{array}$

2.2 Forward Modeling $\quad 18$

$\begin{array}{lll}2.2 .1 & \text { Smoothing } & 19\end{array}$

2.2.2 Principal Component Analysis 21

2.3 Discussion 23

3 Proposed Semiparametric Structural Model 24

3.1 Arbitrage-free Swap Prices 24

3.2 Structural Model for Swap Prices 25

4 Estimation Procedure $\quad 27$

4.1 Computing Arbitrage-Free Prices 27

4.2 Reduced Form Model 28

4.3 Maximum Smoothness 29

4.4 Generalized Maximum Smoothness for Scarce Data Sets 29

5 Case Studies $\quad 31$

5.1 Arbitrage Level Analysis 32

5.2 Elementary Contracts Estimation 35

5.3 Dimensionality Reduction 48

6 Conclusions $\quad 59$

$\begin{array}{lll}7 & \text { Next Steps } & \mathbf{6 0}\end{array}$

$\begin{array}{ll}7.1 & \text { Probabilistic Forecasting }\end{array}$

$\begin{array}{lll}7.2 & \text { Considerations } & 68\end{array}$

$\begin{array}{lr}\text { A Nomenclature } & 69\end{array}$

$\begin{array}{lll}\text { B Descriptive Statistics } & 71\end{array}$ 


\section{List of figures}

Figure 5.1 Histogram of arbitrage levels in the Nordic power market. 33

Figure 5.2 Histogram of arbitrage levels in the Brazilian power market. 33

Figure 5.3 Contracts with the highest arbitrage on the Brazilian's

forward market.

Figure 5.4 Contracts with the highest arbitrage on the Brazilian's forward market and their arbitrage-free prices.

Figure 5.5 Normalized seasonality functions.

Figure 5.6 Estimated residuals on March 2nd 2018 without maximum smoothness criterion in time.

Figure 5.7 Estimated residuals on March 2nd 2018 with maximum smoothness criterion in time.

$\begin{array}{lll}\text { Figure 5.8 } & \mathrm{M}+1 \text { arbitrage-free swaps from BBCE. } & 39\end{array}$

Figure 5.9 $\mathrm{M}+2$ arbitrage-free swaps from BBCE. 40

Figure 5.10 M+3 arbitrage-free swaps from BBCE. 41

Figure 5.11 M+4 arbitrage-free swaps from BBCE. 42

Figure 5.12 M+5 arbitrage-free swaps from BBCE. 43

Figure 5.13 M+6 arbitrage-free swaps from BBCE. 44

Figure 5.14 Q+1 arbitrage-free swaps from BBCE. 45

Figure 5.15 Q+2 arbitrage-free swaps from BBCE. 46

Figure 5.16 Continuous forward curve and arbitrage-free swaps from NordPool.

Figure 5.17 Continuous forward curve and arbitrage-free swaps from $\mathrm{BBCE}$.

Figure 5.18 Maximum maturity reduction between trading days.

Figure 5.19 First three factor loadings from NordPool residual's data set.

Figure 5.20 First three factor loadings from BBCE residual's data set. 50

Figure 5.21 Components from the NordPool data set. The ADF-Test was not able to reject the null hypothesis of existing a unit root $(10 \%, 1 \%, 10 \%$ levels of significance, for each component, respectively).

Figure 5.22 Components from the BBCE data set. The ADF-Test was not able to reject the null hypothesis of existing a unit root for the first two components (5\% and $1 \%$ levels of significance, respectively). In contrast, the null hypothesis was rejected for the third component.

$\begin{array}{lll}\text { Figure 5.23 Fitted level and slope factors for NordPool. } & 53 \\ \text { Figure 5.24 Fitted level, slope and curvature factors for BBCE. } & 54\end{array}$

Figure 5.25 $\mathrm{M}+3$ arbitrage-free swaps from NordPool recovered with 2 components.

Figure 5.26 Q+1 arbitrage-free swaps from NordPool recovered with 2 components.

Figure 5.27 Y+2 arbitrage-free swaps from NordPool recovered with 2 components. 
Figure 5.28 M+1 arbitrage-free swaps from BBCE recovered with 3 components.

Figure 5.29 Q+1 arbitrage-free swaps from BBCE recovered with 3 components.

Figure 7.1 Probabilistic forecasting scheme.

Figure 7.2 Calculation of arbitrage-free prices scenarios.

Figure 7.3 Simulation of Q+7 NordPool prices.

67

Figure 7.4 Simulation of Y+2 NordPool prices. 


\section{List of tables}

Table 5.1 MAPE NordPool (2 components) 55

Table 5.2 MAPE BBCE (3 components) 55

Table 7.1 Probabilistic calibration for monthly swaps from NordPool 64

Table 7.2 Probabilistic calibration for quarterly swaps from NordPool 65

Table 7.3 Probabilistic calibration for yearly swaps from NordPool 66

Table B.1 Descriptive Statistics from NordPool time-series $\quad 71$

Table B.2 Descriptive Statistics from BBCE time-series 72 


\section{Introduction}

Electric power sectors were conceived as vertically integrated industries, with all supply chain levels belonging to the same owner. However, since the 1990s, many countries began a process of vertical and horizontal unbundling, where the competition was gradually inserted in generation and retail while transmission and distribution usually remained as natural monopolies. The deregulating development introduced competitive wholesale markets and power derivatives contracts, such as forwards and futures, to meet the agent's needs on this new paradigm [1]. Therefore, understanding the drivers of spot and derivatives prices and translating this knowledge into an analytical framework is extremely important to the companies' decision-making process entering the electricity markets.

Limited storability and transportability make electricity a peculiar commodity. The former is bounded by reservoir volumes, whose growth slows down due to environmental restrictions, and battery capacities, which remains an economically infeasible solution. On the other hand, transportability is limited by network constraints, such as transmission line capacities and transportation losses [1]. Hence, electricity cannot be "carried" across time and space as other commodities.

Those characteristics are imperative when explaining the behavior of electricity spot and derivative prices. Electricity delivered at different times and locations is perceived as distinct commodities, implying on spot prices becoming dependent on supply and demand levels in every moment and location [1]. Distinguishing weekdays and weekends, on-peak and off-peak hours, and seasonal effects are of great relevance when dealing with power pricing. Moreover, since it's impossible to "carry" electricity over time, some forwardlooking information only affects a part of the forward curve. This echoes in forward prices of different maturities having much lower correlation when compared to other commodities. Also, as electricity cannot be economically saved for future use, short-term contracts are not adequate for hedging long-term risks in electricity markets [2], [3].

Another particularity of electricity is that it is a "flow commodity". This reflects on forward and future contracts with a definition of a delivery period 
(e.g., week, month, quarter, year) during which electricity is continuously delivered, in contrast to other commodities whose derivatives are based on a single delivery in a fixed date in the future. For this reason, electricity contracts are usually referred to as swaps. Consequently, electricity forward curves are composed of swaps with different delivery periods, resulting in an overlapped structure. In this scope, two main approaches are applied to pricing derivatives, including those in power markets. They are referred to as spot and forward modeling.

Spot based methods define an analytical expression for the dynamics of spot prices, and then a closed-form solution for forward prices are obtained by some form of no-arbitrage conditions. The most representative framework in this line of study is the Schwartz-Smith model, initially conceived for single-delivery commodities. Its structure was adapted in [1] to incorporate predictable components, such as seasonality and weekly patterns, while [4] assimilated a jump-diffusion method to deal with spikes observed in electricity spot prices. This was also addressed in [5], [6], [7], [8] and [9].

In contrast, forward based methods delineate the forward curve's stochastic representation directly and are usually conceived under the Heath-JarrowMorton (HJM) framework, originated in the fixed income market. The HJM scheme is built under the premise of instantaneous forward prices, which means its application to electricity contracts is not straightforward. Its dynamics are limited by a stochastic process governed by independent Brownian motions associated with volatility terms. Therefore, assuming a Gaussian distribution is intrinsic to its preliminary definition. Two distinct ways to develop HJM models for power markets were suggested in [10]: the first disregards overlapped contracts, i.e., only a subset of traded swaps with mutually exclusive delivery periods are considered, since the no-arbitrage condition is no longer an issue. The second and most used alternative involves applying HJM on estimated electricity forward prices, named in our study as elementary forward contracts. Those derivatives are not observed in the electricity market and must be derived by smoothing techniques.

The computation of elementary forward contracts is usually based on the maximum smoothness criterion, originated in fixed income markets [11]. It aims to find the forward prices that reconstruct the swap contracts with the characteristic of being the smoothest function over the maturities under analysis. The two most fundamental formulations observed in the literature of electricity pricing comes from [12] and [10]. In the former, a bottom-up model estimates prices used to delineate the seasonality observed in power markets. Then, a bi-objective quadratic optimization problem is defined, where 
the squared errors between the elementary prices and the results from the bottom-up model are minimized, in addition to the total curvature of the nonparametric representation of elementary contracts (maximum smoothness). The optimized prices are constrained by bid and ask observed in the market.

The methodology proposed in [10] described elementary prices as a sum of a seasonality function and a residual term. Different from [12], the maximum smoothness criterion was imposed on the residuals instead of directly on forward prices to better retain seasonal patterns. Polynomial splines of order four parameterized the residuals. Two different optimization problems were conceived, differing only in the constraint that relates the optimized and observed prices: the first is analogous to the one in [12], where prices were constrained by bid and ask levels; the second matched the optimized swaps with closing prices.

Following the smoothing process, Principal Component Analysis (PCA) is usually executed on a data set computed from elementary forward prices to help determine the number $\mathrm{K}$ of Brownian motions and the shape of volatility terms, necessary for practical applications of HJM. PCA was performed in [2] on two sets built from elementary prices from NordPool, and three components explained $80 \%-83 \%$ of the total variance, depending on whether it refers to price differences or returns. In [10], PCA was also performed on a data set from more updated NordPool swap prices, and the authors found similar conclusions, with three factors being able to explain $70 \%$ of the total variance of log returns. Both studies reported that ten factors were needed to describe $95 \%$ of the total variance. Those values are significantly lower when compared to the ones from the US copper market in [13]. Since PCA is a step in applying HJM on electricity swap prices, the dimensionality reduction must be done under a data set coherent to the problem formulation. As we will show later, this condition might hide higher percentages of total variance explanation.

In this context, we propose a novel semiparametric structural model for electricity forward curves. The semiparametric nomenclature refers to the representation of elementary forward contracts as a sum of a parametric term, that explains the price behavior, and a non-parametric model for the error. From the parametric side, our framework is flexible as it allows representing elementary price linearly dependent on seasonal effects as well as other explanatory variables. From the non-parametric side, our framework fits the scope of smoothing techniques, but with the novelty of considering maximum smoothness both in maturity and time dimensions. For estimation purposes, a reduced-form model is derived by embedding the parametric structure of the elementary contract into the non-arbitrage relationships with the observed 
swap prices. Different from [12] and [10], the expression that relates swaps and elementary contracts is written in terms of time to maturity and not delivery dates. Besides being imperative when performing data analysis, describing contracts in terms of time to maturity allows us to introduce the smoothing in the time dimension and not only in maturity, as is done in the literature. This is important when dealing with low-liquidity markets, as will be discussed later in this work.

Furthermore, instead of constraining the optimized prices within the bid and ask limits or fitting it perfectly with observed swaps, we allow a mismatch between estimated and observed prices. This is crucial to completely assimilate overlapped contracts, which is an inherent condition in flow commodities. All smoothing techniques mentioned so far proceed after manipulating the original set of settled assets to bypass infeasibilities that could occur otherwise. In our proposed framework, overlaps are not an issue and are essential in estimating elementary forward prices. Also, the mismatch has an interesting interpretation of arbitrage opportunities in the market.

The estimation procedure of elementary prices consists of a hierarchical approach, where the non-arbitrage condition is prioritized. Then, the model's parameters are estimated, followed by the computation of elementary contracts. In this last stage, two formulations were proposed: one with the maximum smoothness only on the maturity dimension and the other with the incorporation of smoothness in time to help when dealing with scarce data sets. Here, the maximum smoothness criterion is applied to the residual term, following [10].

Principal component analysis of the smoothed residuals generated impressive results. We were able to explain more than $97 \%$ of total variance with only three components and interpretable factor loading shapes for the Brazilian and Nordic power markets. These values stand out from those found in the literature.

The remainder of this dissertation is organized as follows. Chapter 2 presents a literature review on the most used methods in modeling electricity derivatives. Chapter 3 presents our novel semiparametric structural model, followed by the development of the hierarchical estimation procedure for elementary forward contracts in Chapter 4 . Chapter 5 refers to the applications of our framework in the Brazilian and Nordic power markets. Finally, Chapter 6 presents the conclusions while Chapter 7 defines our next steps, focusing on the extension of our framework for probabilistic forecasting purposes. 


\section{1 \\ Objective and Contributions}

In this work, we develop a semiparametric structural model for electricity forward curves that acknowledges forward prices drivers, such as seasonality and any related explanatory variable, and its smooth behavior through time. We are able to obtain a continuous daily forward curve that makes use of overlaps through a hierarchical estimation procedure with three main steps: computation of arbitrage-free swap prices, parameters estimation and, finally, the gathering of elementary forward prices. We extend the maximum smoothness criterion to address scarce data challenges in low-liquidity markets. Finally, we present a dimensionality reduction step based on principal components analysis, which is a more suitable approach for forecasting purposes. Thefefore, our main contributions are:

1. Establish a flexible semi-parametric representation for elementary forward prices, where any seasonal structure or exogenous variables can be linearly treated;

2. Account for arbitrage opportunities in the power market and use this information as an outlier detector;

3. A hierarchical estimation procedure able to handle scarce data sets;

4. We show that more than $97 \%$ of the residuals variability can be explained by only three principal components for both the Brazilian (BBCE), 98.4\%, and Nordic (NordPool), 97.4\%, electricity markets. It is important to note that previous studies show that at least 10 components are needed to explain $95 \%$ of the total variance. 


\section{2}

\section{Literature Review}

The literature review will be divided into two methodologies that we refer to as spot and forward modeling. While the forward modeling directly characterize curve dynamics, the spot modeling approach specify a process for spot prices and then, under simplifying assumptions, derive forward and future price dynamics based on no-arbitrage principles. These two are the main approaches when dealing with derivatives pricing, in particular of electricity markets [14].

\section{1}

\section{Spot Modeling}

Spot based methods define an analytical expression for spot price dynamics, and then a closed-form solution for forward prices is usually obtained by non-arbitrage conditions. The most common studies in this research line refer to the Schwartz-Smith framework [15], conceived initially for single-delivery commodities, e.g., oil future prices. The authors define the spot logarithm dynamics as a sum of two stochastic factors defining the short-term deviations in prices (Ornstein-Uhlenebck process) and the equilibrium price levels (Brownian motion process). After that, the forward prices are assumed to follow the conditional expectation of spot prices in the contract's delivery dates. Applications of the Schwartz-Smith framework in the electricity market mostly vary according to the number of factors considered, the inclusion of seasonal patterns, and the treatment of price spikes.

In [1], the authors developed one and two-factor models based on the Schwartz-Smith framework for the NordPool market. They evaluated the importance of the predictable components in electricity spot prices and how they reflect on derivative valuation. This is important due to the limited storability of electricity, where the current spot level is not a good approximation for understanding the long-term price dynamics. Instead, we should estimate the expected value of spot prices during the swap's delivery period and, thus, the systematic comportment of spot prices through time is indispensable. The assessed models differ with respect to the stochastic factors assumed for the spot price dynamics and the deterministic function, representing the predictable 
components. The latter was divided into two terms: the first addressed the price level differences between weekdays and weekends through a dummy variable; the second characterized seasonality with a cosine function or dummies for each month. Since this was an early study, insufficient data was an issue. Therefore, the estimation of seasonal adjustment parameters used historical spot price data and of the remaining parameters, the available forward prices.

While [1] accounted for the mean-reverting nature of electricity, they failed to report the price spikes inherent to the electricity sector. Since the underlying commodity cannot be economically stored, electricity bought in the spot market must be used almost immediately once purchased. Typical hedging strategies that involve holding specific amounts of the commodity are not possible. In addition, low elasticity in the short-term demand also contributes to price spikes. [4] aims to address the limitations of the previous study by capturing mean reversion, jumps, and seasonality. The model's parameters were also estimated with a hybrid approach using both spot data and current forward prices of England and Wales markets. The former was used to calculate the seasonality component, the rolling historical volatility, the mean reversion rate, and the jumps' frequency and standard deviation. The latter was applied in the market price of risk estimation. Logarithmic returns composed the data set, and a Fourier series of order five described the seasonal component. The authors concluded that the evidence of fat tails in the distribution of electricity returns, resulting from spikes and time-varying volatilities, indicates a possibility of departing from the Gaussian assumption in such models.

Other studies intended to face the price spikes in the electricity spot series. In [5], three different mean-reverting jump-diffusion models for the volatile behavior of power prices were proposed by incorporating either multiple jumps, regime-switching, or stochastic volatility. [6] suggested using mean reversion coupled with upward and downward jumps, with the direction of the jumps depending on the price level. So, the chances of a negative jump being followed by a positive jump and vice-versa are higher. The same was defended by [7] and [8]. [6] aimed to find the best model for kurtosis by filtering raw price data using different thresholds and selecting the one leading to the best-calibrated kurtosis.

Studies incorporating jump-diffusion methods usually writes the underlying stochastic variable as a sum of a mean reversion drift term, a constant or time-dependent volatility term, and a pure jump process, modeled by a homogeneous Poisson distribution with a constant intensity $\lambda[16]$. However, this is an unrealistic representation as the probability of price spikes differs between different seasons (spike clustering). To bypass this drawback, researchers used 
Markov Regime-Switching (MRS), representing how the stochastic variables follow multiple processes or regimes, switching between them according to an unobserved Markov chain. A class of general models composed of mean reversion, seasonal adjustment, time-varying volatility, and jumps were developed in [9]. The models were tested in different markets, such as Argentina, Australia, Canada, New Zeland, Netherlands, NordPool, Spain, and PJM. Nevertheless, their goal was to model just the spot prices and not present a closed-form solution for forward prices.

In [3], no stochastic process was considered for electricity spot prices. Instead, the authors wrote the spot price's logarithm as a linear combination of the logarithmic forward prices of fundamentals, such as fuel, emission allowances, and imported electricity. Hence, forward prices are the rational expectation about the spot price at the delivery time, discounted with the riskfree interest rate and risk premium, described as a function of time to maturity. Weekly swap prices from NordPool, with the duration of one year and time to maturity greater than one year were modeled. The authors demonstrated that the forward price dynamics differ from the spot prices when the delivery is considerably far. As the delivery period approaches, long-term and spot prices become more similar. All variables (electricity and fundamental's forward prices) were considered endogenous and function of their past values and past values of the other variables on a Vector Autoregressive Model (VAR) with a lag equal to two. Initially, there was some remaining residual autocorrelation structure. The authors found out that aluminum prices were a determinant factor in electricity prices during the period under analysis, being added to the framework. Also, auxiliary dummies accounted for shocks. Both interferences helped improve the results, and two stationary long-run relationships were found. Moreover, the risk premium dynamics in the long-term electricity forward from NordPool is relatively low and could be considered constant.

\section{2}

\section{Forward Modeling}

Studies under the forward modeling paradigm are usually based on the Heath-Jarrow-Morton (HJM) framework [17] originated in the fixed income market. It directly models the evolution of instantaneous forward rates given an initial condition and a stochastic process for its subsequent movements, considering no-arbitrage assumptions. HJM formulations are characterized as multifactor models. A number of $\mathrm{K}$ independent factors, represented by Brownian motions, determine the dynamics of the entire forward curve and are associated with a volatility term commonly written as a function of the trading 
and maturity dates. It is mandatory for practical applications to determine the number K of factors and the shape of the volatility terms. Principal Component Analysis (PCA) plays an essential role in this process, which will be highlighted later in this chapter.

The HJM framework is built under the premise of instantaneous forward price, which means its application to electricity contracts is not straightforward. When modeling swap settlements, specific conditions must be satisfied to guarantee an arbitrage-free dynamic [10]. For instance, in a power market as NordPool, swaps with the delivery period of a week, month, quarter, and year are available. One could buy three monthly contracts, exactly covering a quarterly asset. Furthermore, a swap to be delivered during a year could also correspond to four quarterly contracts for the same year. This clearly emphasizes how the products must be connected to avoid arbitrage opportunities.

In [10], the authors discussed how the direct implementation of HJM in swap prices leads to intractable models. This is due to the non-arbitrage relations exemplified previously, since a swap contract defined for a specific delivery period yields the same payoff of a portfolio of smaller swaps with their delivery period resulting from the former's decomposition. To circumvent those obstacles, the authors suggested two different approaches to develop HJM models for electricity prices. The first disregards overlapped contracts, i.e., the HJM framework only considers a subset of traded swaps with mutually exclusive delivery period. Therefore, no-arbitrage condition is no longer an issue. The second approach involves applying HJM on estimated electricity forward prices, named in our study as elementary forward contracts. Those derivatives are not observed in the electricity market and must be derived by smoothing techniques that estimate the underlying assets based on the negotiated and available prices. This is the most common tactic used by the authors in forward modeling, but it is essential to be aware that the estimated data is intrinsically dependent on the implemented algorithm.

Hence, the remainder of the literature review regarding the forward modeling paradigm is divided into two parts: the first analyses the smoothing techniques' current status. The second shows how the elementary forward prices are considered in the HJM framework, highlighting the results of Principal Component Analysis.

\subsection{1 \\ Smoothing}

The calculation of elementary forward contracts is usually a result of the maximum smoothness criterion. The concept was first used in fixed income 
markets by [11], and it aims to find the forward prices that reconstruct the swap prices with the characteristic of being the smoothest function over the maturities under analysis. A curve's smoothness is related to its curvature, which means that these criterion' objective function is to minimize the total slope variation across maturities. Due to the intense seasonal patterns of electricity prices, this technique's application in this context is coupled with a seasonal adjustment step to address the particularities of the underlying commodity.

In [12], the authors modeled high-resolution forward price curves by combining information of the observed bid and ask prices with information from forecasts generated by bottom-up models. The calculation of elementary forward contracts was obtained by a quadratic bi-objective optimization problem. The first term aims to minimize the difference between the forward prices and the bottom-up model results, while the second refers to the maximum smoothness criterion and is weighted by a value $\lambda$. Here, bottom-up outcomes try to reproduce the seasonal behavior of electricity prices. In addition, the prices recovered by elementary contracts are constrained by the bid and ask levels observed in the NordPool market. If the parameter $\lambda=0$, no smoothing is performed. However, if $\lambda \rightarrow \infty$, the shape of the seasonality of electricity prices is indifferent. The framework quality was evaluated by its ability to price nontraded maturities compared to a model with only the maximum smoothness criterion and to a simple functional relationship along the maturity dimension written as a truncated Fourier series. The proposed model performed better than the suggested benchmarks.

The smoothing techniques developed in [10] described elementary prices as a sum of a seasonality component and an error term. Different from [12], the maximum smoothness criterion was imposed on the residuals instead of directly on forward prices to retain seasonal patterns better. Polynomial splines of order four parameterized the residuals. Two different optimization problems were conceived, differing only in the constraint that relates the optimized and observed prices: the first is analogous to the one in [12], where prices were constrained by bid and ask levels; the second matched the optimized swaps with closing prices.

The seasonality is an exogenous prior function. Its parameters are fitted using the least square approach, usually to reproduce the seasonal dynamics of spot prices. The spline specification and the maximum smoothness for residuals ensure the fit to the market's observed prices. The authors tested the effect of the choice of seasonal functions on the smoothed forward curve for the NordPool data set, using the formulation with the constraint of the 
bid/ask boundaries. Three different specifications were tested: no seasonality, a trigonometric function fitted into spot prices [1], and spot prognosis from a bottom-up model, similar to [12]. The impact of the distinct specifications on the shape of the elementary forward curve varies with maturity: all seasonality functions resulted in similar forward curves for maturities until one year; in contrast, longer maturities are strongly affected by the shape adopted, and incorporating seasonality becomes imperative to outline the estimated forward prices.

After computing the elementary forward contracts, they suggested two ways of constructing historical prices that have no overlaps: one of fixeddelivery and other of average-based forwards. The latter is composed of weighted averages between the elementary contracts composing the swaps' delivery period. For instance, suppose a weekly contract whose delivery begins in 7 and ends in 14 days. The correspondent average-based price will be the weighted average between elementary forwards with maturities between 7 and 14 days. To guarantee no overlaps, they chose each swap's beginning date as the next day after the ending of the previous contracts. For the fixeddelivery forwards, their maturities are chosen as mid-points of the averagebased forwards.

In [18], the authors proposed a novel method based on a constrained least square optimization procedure where the level of futures and historical spot prices are simultaneously taken into account in a joint optimization approach. The elementary prices are modeled by one trigonometric spline, which accounts for both seasonality and residual terms. For a more in-depth analysis of the proposed method, refer to [18].

Smoothing techniques are important to price unobserved elementary forward contracts and extract information about the volatility behavior of prices, which will be discussed next. Evaluating the results from those techniques is not straightforward since there are no observed elementary prices to be directly compared to. Furthermore, the estimated prices depend on the optimization framework's assumptions, such as a parametric representation for the residuals, the chosen seasonality shape, and the application of maximum smoothness criterion directly on forward prices or on residuals. Therefore, the smoothing framework should be assessed according to its application goal.

\subsection{2}

\section{Principal Component Analysis}

As mentioned previously, most applications on forward modeling based on HJM perform some variation of the maximum smoothness criterion to 
calculate elementary forward contracts, which have no overlaps and can deal with some of the swaps implications. Following this step, PCA is usually executed to find common factors that explain the variance of the data under analysis. Therefore, the two pieces of information necessary on a forward modeling framework can be determined: the number of factors $\mathrm{K}$ is defined based on the percentage of variance explained by the components, and the shape of the volatility terms associated to the Brownian motions are inferred from the factor loadings.

In [2], the authors performed principal component analysis on two sets built from the smoothest function that prices all traded assets within the bid/ask spread of the NordPool market. The authors created fixed-delivery sets on both price differences and price returns. The analysis comprehended 21 vertices, 18 of them representing maturities up to one year, and three in the second year. Some interesting statistical analyses showed that the standard deviation sharply falls with time to maturity, which is intuitive since the curve's short-term is more affected by spot price variations. The inadequacy of Gaussian distributions was also highlighted due to the high kurtosis and positive skewness observed in the data set.

The first component from PCA explained between $68 \%-70 \%$ of the total variance, depending on whether it refers to price differences or returns. Adding one more factor increased the percentage to $75 \%-78 \%$ while incorporating a third allowed the explanation of $80 \%-83 \%$. The authors acknowledged that, to account for $95 \%$ of total variability, more than ten factors would be necessary. Those values are significantly lower when compared to those obtained from the US copper market in [13]. Due to the limited storability of electricity, the term structure common to all maturities represents $75 \%$ of the total variance, while the remaining $25 \%$ are specific to each maturity. The first two factors responsible for the dynamics underlying all curve have well-know behaviors in the literature. The first factor falls as the maturity increases and stabilized after one year (level factor), and the second presents different signals between the short and long ends of the curve (slope or steepness factor).

In [10], the authors extended the previous work by performing PCA on an average-based data set from more updated NordPool swap prices. Their conclusions were similar to those from [2], with three factors being able to explain $70 \%$ of the total variance of log returns. Ten factors were also needed to describe $95 \%$ of the total variance. A different analysis was made regarding the separate evaluation of individual market segments (weekly, monthly, quarterly, and yearly swaps). Three factors explained a higher share of total volatility for each sector ( $96 \%$ for monthly, $80 \%$ for quarterly, and $100 \%$ for yearly swaps). 
Furthermore, the factor loadings suggested the three typical level, steepness, and curvature profiles observed in interest rates, promoting an interpretability quality. Finally, examining the correlation matrix of normalized electricity future prices returns, the authors concluded that contracts with different delivery periods have correlations lower than those close together.

\section{3}

\section{Discussion}

Spot based methods allow for an intuitive interpretation of the electricity forward curve dynamics. They have the flexibility to account for a hybrid estimation, where historical spot and forward prices might be applied when calculating the model's parameters since an explicit relation between both markets is the core of this methodology. Inputting observed characteristics of electricity spot prices is also straightforward, as well as their theoretical interpretation. Nonetheless, its advantage is also a drawback. Although spot prices can be easily manipulated through its mathematical equation, factors affecting only forward prices are not as easy to be included.

In contrast, forward modeling allows the direct description of forward price dynamics without specifying an explicit relationship with spot prices. Nevertheless, it depends on a higher amount of traded prices on the market because of this approach. Also, since HJM is built under the premise of instantaneous forward contracts, it is necessary to disregard overlaps or build a new data set composed by smoothed elementary prices. Although the forward curve is directly portrayed, incorporating exogenous structures on the HJM representation might not be straightforward.

Both Schwartz-Smith and HJM, which inspires most spot and forward modeling, respectively, assume normality in some step of its development. However, empirical results show excess kurtosis and positive skewness on electricity prices, indicating that Gaussian distributions are not the most suitable choice to represent them. 


\section{3 \\ Proposed Semiparametric Structural Model}

In this work, the proposed framework is based on two mathematical representations governing pricing dynamics. Therefore, this section starts with the algebraic characterization of arbitrage-free swap prices followed by the structural model for swap prices.

\section{1}

\section{Arbitrage-free Swap Prices}

Elementary forward contracts are unobserved derivatives of the same granularity and, as a consequence, without overlaps. A more intuitive understanding is to interpret them as smaller pieces of the original swap, resulted from the breaking of the delivery period into shorter fragments.

In a competitive power market, negotiate a swap contract, defined by a continuous delivery period, should be equivalent to trade multiple elementary contracts for the same interval of time, i.e., the arbitrage opportunities should be close to zero. Equation (3-1) defines the arbitrage net present value (NPV):

$$
\Delta_{t, i}=\sum_{j=h_{t, i}}^{h_{t, i}+\Delta T_{t, i}} \frac{F_{t, i}-f_{t, j}}{(1+r)^{j}}, \quad \forall t \in \mathcal{T}, \forall i \in \mathcal{N}_{t}
$$

Where $f_{t, j}$ is the price of the daily elementary forward contract (not observed in the market) on the date $t$, to be delivered $j$ days ahead . $F_{t, i}$ is the observed price of the swap $i$ on the same date $t$ and the interval $\left[h_{t, i}, h_{t, i}+\Delta T_{t, i}\right]$ set its delivery period.

The non-arbitrage condition is the most important premise adopted on financial models, and it establishes that agents shouldn't be able to make a risk-free profit. In a market with the absence of arbitrage, the value of $\Delta_{t, i}$ would be zero, but that is unrealistic. A simple manner of handling arbitragefree swap prices consists of modeling only contracts without overlap. However, as we'll show in the next subsection, elementary forward contracts are state variables inferred from the settled swap prices. Therefore, the intersection between delivery periods would be relevant information in the estimation 
procedure. Hence, instead of disregarding overlaps, our arbitrage-free swap prices are obtained by opening the summation in (3-1) and writing $F_{t, i}$ as:

$$
F_{t, i}=\sum_{j=h_{t, i}}^{h_{t, i}+\Delta T_{t, i}} f_{t, j} \frac{(1+r)^{-j}}{J_{t, i}}+\zeta_{t, i}, \quad \forall t \in \mathcal{T}, \forall i \in \mathcal{N}_{t}
$$

where $J_{t, i}=\sum_{j=h_{t, i}}^{h_{t, i}+\Delta T_{t, i}}(1+r)^{-j}$ and $\zeta_{t, i}=\frac{\Delta_{t, i}}{J_{t, i}}$ is the arbitrage level in prices.

Expression (3-2) highlights how swaps are the sum between a portfolio of elementary contracts for its delivery period, which are the arbitrage-free swap prices, and the arbitrage levels. In addition, it's easy to notice the absence of arbitrage when there are no overlaps, since each $F_{t, i}$ can be written as a linear combination of different sets of elementary contracts.

\section{2}

\section{Structural Model for Swap Prices}

Structural models are time series frameworks that acknowledges their dynamic evolution through time. This flexibility is related to the presence of unobserved variables, called state variables, that act as time-varying coefficients and are estimated from the series under analysis. The definition of a structural model is based on a state space scheme, which in our proposed framework is described as:

$$
\begin{array}{ll}
F_{t, i}=\sum_{j=h_{t, i}}^{h_{t, i}+\Delta T_{t, i}} f_{t, j} \frac{(1+r)^{-j}}{J_{t, i}}+\zeta_{t, i}, & \forall t \in \mathcal{T}, \forall i \in \mathcal{N}_{t} \\
f_{t, j}=\boldsymbol{x}_{t, j}^{T} \boldsymbol{\beta}+\varepsilon(t, j), & \forall t \in \mathcal{T}, \forall j \in \mathcal{J} .
\end{array}
$$

Expression (3-3) is the measurement equation and translates the relation between the modelled time series $\left(F_{t, i}\right)$ and state variables $\left(f_{t, j}\right)$. (3-4) is the transition/state equation, where the time evolution of the state variable is defined. It is important to say that usual state space models also have simple error term. Here, $\zeta_{t, i}=\frac{\Delta_{t, i}}{J_{t, i}}$ is a weighted error accounting for the present value discount factor within each contract delivery period. Furthermore, in the expression of $f_{t, j}$, the residual $\varepsilon(t, j)$ is a function of time and maturity, and it is not the regular uncorrelated errors considered in state space models. In this study, we do not assume a parametric form for $\varepsilon(t, j)$. Rather, we assume that function $\varepsilon$ belongs to the set, $\mathcal{S}$, of smooth functions.

Equation (3-4) characterizes the dynamics of elementary forward prices. Besides the residuals $\varepsilon(t, j)$, any necessary structure could be inserted in the 
vector $\boldsymbol{x}_{t, j}$ : calendar effects, such as the impact of weekdays and weekends on prices; seasonality through dummy variables or sine and cosine functions; trends; and exogenous variables. Regarding the latter, it is crucial to have a powerful statistical model able to generate scenarios for both the estimation and probability forecasting steps. The vector $\boldsymbol{\beta}$ defines the associated coefficients to be estimated. 


\section{4}

\section{Estimation Procedure}

The estimation procedure defined in this chapter aims to calculate the elementary forward prices induced by swap contracts traded on electricity markets. The proposed semiparametric structural model presented so far is based on the relationship between the swap prices $F_{t, i}$ and the elementary contracts, $f_{t, j}$. To address the challenge of estimating a semiparametric structural model, we propose a hierarchical approach based on the following steps. We first estimate arbitrage-free prices $Y_{t, i}$ to prevent that the next steps artificially play with arbitrage levels through time to better adjust the parametric and nonparametric parts of the model. Then, we derive a reduced form to estimate the parametric part of the model, $\boldsymbol{\beta}$. With fixed values of $\boldsymbol{\beta}$, we estimate the nonparametric residuals, $\varepsilon(t, j)$, via maximum smoothness in time and maturity dimensions.

Additionally, it's reasonable to argue that, if $\zeta_{t, i}$ has a high absolute value, the correspondent settled price is a strong candidate of abnormality, as it diverges too much from the average behavior. If an arbitrage threshold is pre-defined, swap prices $F_{t, i}$ with an associated $\zeta_{t, i}$ higher than the established limit could be disregarded.

The following subsections detail the steps mentioned above.

\section{1}

\section{Computing Arbitrage-Free Prices}

Let arbitrage-free prices be:

$$
Y_{t, i}=F_{t, i}-\zeta_{t, i}, \quad \forall t \in \mathcal{T}, \forall i \in \mathcal{N}_{t}
$$

To compute $Y_{t, i}$, we must calculate the values of $\zeta_{t, i}$. To do that, we use the following optimization problem for each trading date:

$$
\begin{aligned}
& \min _{\zeta, f} \theta \\
& \text { s.t. } F_{t, i}=\sum_{j=h_{t, i}}^{h_{t, i}+\Delta T_{t, i}} f_{t, j} \frac{(1+r)^{-j}}{J_{t, i}}+\zeta_{t, i}, \quad \forall i \in \mathcal{N}_{t} \\
& \theta \geq\left|\zeta_{t, i}\right|, \quad \forall i \in \hat{\mathcal{N}}_{t},: \gamma_{i},
\end{aligned}
$$


which aims to minimize the infinity norm of $\boldsymbol{\zeta}_{t}$. To avoid degeneracy (multiple solutions for $\boldsymbol{\zeta}$ ) in the infinity-norm minimization, we propose the iterative process:

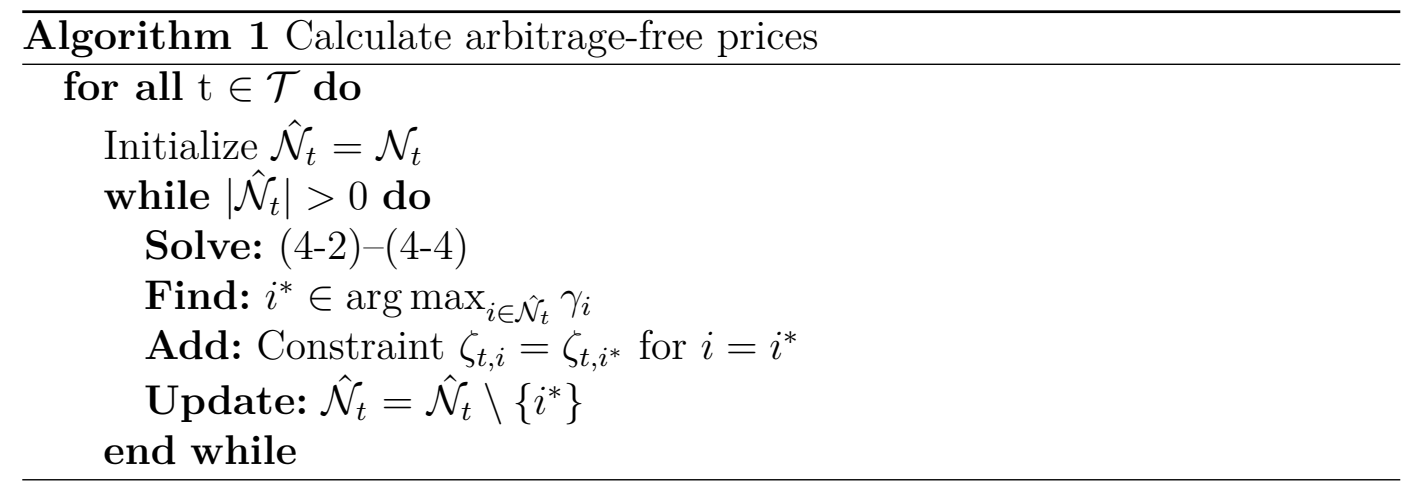

The infinity norm is justified on the need to assess minimum level of arbitrage opportunities before estimating the model.

\section{2}

\section{Reduced Form Model}

Embedding (4-1) and (3-4) in (3-3), we obtain the following reduced form model:

$$
Y_{t, i}=\boldsymbol{X}_{t, i} \boldsymbol{\beta}+\eta_{t, i}, \quad \forall t \in \mathcal{T}, \forall i \in \mathcal{N}_{t},
$$

where,

$$
\begin{aligned}
\boldsymbol{X}_{t, i} & =\sum_{j=h_{t, i}}^{h_{t, i}+\Delta T_{t, i}} \boldsymbol{x}_{t, j}^{T} \frac{(1+r)^{-j}}{J_{t, i}} \\
\eta_{t, i} & =\sum_{j=h_{t, i}}^{h_{t, i}+\Delta T_{t, i}} \varepsilon(t, j) \frac{(1+r)^{-j}}{J_{t, i}}
\end{aligned}
$$

Equation (4-5) illustrates how the reduced-form model becomes as simple as a linear regression. $\boldsymbol{X}_{t, i}$ is a vector with the weighted average of each independent variable during the swap delivery period. They are deterministic values for each trading date $t$ and contract $i$. The error term $\eta_{t, i}$ refers to the parcel of the swap price not explained by the structure introduced on the elementary forwards. They are a weighted average of the residuals $\varepsilon(t, j)$ during the swap delivery period.

The calculation of coefficients $\boldsymbol{\beta}$ is directly related to the error term, $\eta_{t, i}$, as observed in (4-5). Therefore, the values of $\boldsymbol{\beta}$ are estimated by the ordinary 
least square (OLS) problem, as given by:

$$
\boldsymbol{\beta}^{*} \in \arg \min _{\boldsymbol{\beta}} \sum_{t \in \mathcal{T}} \sum_{i \in \mathcal{I}}\left(Y_{t, i}-\boldsymbol{X}_{t, i} \boldsymbol{\beta}\right)^{2} .
$$

\section{3}

\section{Maximum Smoothness}

In the last stage of our estimation procedure, we estimate the nonparametric part of our model for fixed values of $\boldsymbol{\beta}^{*}$. This requires the definition of some measure that drives the choice of the most appropriate function $\varepsilon(t, j)$ to compose the elementary forward contracts.

Previous studies regarding the modeling of electricity forward curves apply the maximum smoothness criterion on the maturity dimension $j$, either directly on the elementary contracts or on their residuals [12], [19]. These criteria aim to minimize the total curvature of a function given by the sum of the second derivatives in the maturity dimension. Assuming that (3-4) specifies elementary prices, [10] states that imposing a smooth behavior on the error term guarantees that the structure of $f_{t, j}$ is better retained. So, among all the smooth functions in $\mathcal{S}$, we select $\varepsilon(t, j)$ resulted from the maximum smoothness assumption. Here, no premise about the parametric representation for $\varepsilon(t, j)$ is made. Instead, we explicitly refer to the elements of its image, hereafter $\varepsilon_{t, j}$. So, we define the maximum smoothness problem used to estimate $\varepsilon_{t, j}$ as follows:

$$
\begin{array}{ll}
\min _{\varepsilon} & \sum_{t \in \mathcal{T}} \sum_{j \in \hat{\mathcal{J}}}\left(\varepsilon_{t, j+1}-2 \varepsilon_{t, j}+\varepsilon_{t, j-1}\right)^{2} \\
\text { s.t. } & Y_{t, i}=\boldsymbol{X}_{t, i} \boldsymbol{\beta}^{*}+\sum_{j=h_{t, i}}^{h_{t, i}+\Delta T_{t, i}} \varepsilon_{t, j} \frac{(1+r)^{-j}}{J_{t, i}}, \quad \forall t \in \mathcal{T}, \forall i \in \mathcal{N}_{t},
\end{array}
$$

where $\hat{\mathcal{J}}=\{2,3, \ldots, J-1\}$. It's worth mentioning that problem (4-9)(4-10) could be solved for each trading period separately, since the vector of coefficients $\boldsymbol{\beta}^{*}$ is fixed.

\section{4}

\section{Generalized Maximum Smoothness for Scarce Data Sets}

As previously mentioned, (4-9)-(4-10) can be decomposed into smaller problems for each trading date. The elementary contracts with maturities that belong to the intervals $\left\{\left[h_{t, i}, h_{t, i}+\Delta T_{t, i}\right]\right\}_{i \in \mathcal{N}_{t}}$, i.e., the delivery period of the negotiated contracts, will be based on both the swap price level and the 
maximum smoothness criterion. Nevertheless, scarce data can jeopardized the maximum smoothness process presented before.

To clarify this aspect, assume that, on a trading date of August/20, monthly swaps with delivery for September/20 and November/20 were traded. As the objective function (4-9) is defined for all maturities $j \in \hat{\mathcal{J}}$, it's imperative to calculate the residuals $\varepsilon_{t, j}$ that will compose the swap for October/20, which has no settled price defined but links both negotiated contracts. In those cases, when there are no prices to support the choice of $\varepsilon_{t, j}$ and, consequently, $f_{t, j}$, the maximum smoothness criterion connects the maturities that constitute the available and missing swaps. This highlights one relevant feature of our framework, it generates an interpolation effect.

Now, consider a more critical situation. Suppose that only the monthly contract for September/20 was traded on a date $t$, and we want to infer the prices that would have been settled for October/20 and November/20. As the objective function minimizes the slope variation on the maturity dimension, the residuals $\varepsilon_{t, j}$ for $j>h_{t, i}+\Delta T_{t, i}$, where $i$ is the swap for September/20, will be estimated to maintain the final slope of the maturities composing the traded contract. This means that, if $\varepsilon_{t, h_{t, i}+\Delta T_{t, i}}$ increases, decrease or persist the same, this trend will be kept indefinitely for all higher maturities.

In similar cases, it's reasonable to assume that the information of the forward curves for adjacent periods (the days before and after) could be used to interpolate the elementary prices in the presence of missing data. This is especially important when dealing with data from low liquidity markets, such as Brazil. Therefore, we reformulate (4-9)-(4-10) as a bi-objective optimization problem that minimizes the linear combination of smoothing over time and maturity.

$$
\begin{aligned}
& \min _{\varepsilon} \lambda_{1}\left[\sum_{t \in \mathcal{T}} \sum_{j \in \mathcal{\mathcal { J }}}\left(\varepsilon_{t, j+1}-2 \varepsilon_{t, j}+\varepsilon_{t, j-1}\right)^{2}\right]+ \\
& \lambda_{2}\left[\sum_{t \in \mathcal{\mathcal { T }}} \sum_{j \in \mathcal{J}}\left(\varepsilon_{t+1, j}-2 \varepsilon_{t, j}+\varepsilon_{t-1, j}\right)^{2}\right] \\
& \text { s.t. } \quad Y_{t, i}=\boldsymbol{X}_{t, i} \boldsymbol{\beta}^{*}+\sum_{j=h_{t, i}}^{h_{t, i}+\Delta T_{t, i}} \varepsilon_{t, j} \frac{(1+r)^{-j}}{J_{t, i}}, \quad \forall t \in \mathcal{T}, i \in \mathcal{N}_{t}
\end{aligned}
$$

The set $\hat{\mathcal{T}}$ is equal to $\{2,3, \ldots, T-1\}$. Parameters $\lambda_{1}$ and $\lambda_{2}$ must be fixed a priori. If $\lambda_{2}=0$, solving (4-11)-(4-12) is equal to (4-9)-(4-10). 


\section{Case Studies}

In this chapter, we present two case studies that illustrate our structural model's estimation. To highlight the contributions of our work, we consider the traded assets of two different power markets:

1. Nordic (NordPool): Its power market is consolidated and, therefore, is analyzed in multiple studies regarding electricity forward curve modeling [2][12][19]. We've contemplated $\mathrm{M}+1, \ldots, \mathrm{M}+6, \mathrm{Q}+1, \ldots, \mathrm{Q}+8, \mathrm{Y}+1, \mathrm{Y}+2$ and $Y+3$ closing prices from 2013 to 2018. In the $Y+1$ time series, six trading days with prices equal to zero were observed. We've assumed that those negotiations did not occur since these are unreasonable prices. Because it represents less than $0.4 \%$ of the number of observations, scarce data is not an issue when dealing with this set.

2. Brazil (BBCE): The trading on the futures market of Brazil's power sector is really incipient. Changes have been discussed over the last few years, such as incorporating a short-term price with higher granularity and the availability of financial contracts. Still, the market, for now, remains with low liquidity. This implies a considerable amount of missing data, especially for long term settlements, illustrated in our results. The data set has $\mathrm{M}+1, \ldots, \mathrm{M}+6, \mathrm{Q}+1$, and $\mathrm{Q}+2$ assets from 2018 to 2019, and, instead of closing prices, we use the median as a metric to calculate the corresponding daily prices.

The platform for forward electricity trading in Brazil, referred to as $\mathrm{BBCE}$, deals with centralized and decentralized agreements. In the latter, agents define their prices and quantities outside of the platform but use their standardized contracts to delineate the negotiation formally. The prices obtained by the decentralized transactions usually come with a considerable amount of outliers and, thus, they were disregarded in our study.

Recalling the mathematical definition of elementary forward prices in (3-4), to perform our estimation procedure, we must first specify their structure besides the residuals. For the following results, seasonality on the delivery date was included through sine and cosine functions, specifically a truncated Fourier series with only one harmonic. We've chosen this approach as it's a smooth 
approximation of the monthly seasonality that would be obtained by dummy variables. This also directly impacts the forward curve shape, which otherwise would have notorious level shifts between months. Mathematically:

$$
\begin{aligned}
f_{t, j}=\mu & +\beta^{\sin } \sin \left[\frac{2 \pi(t+j)}{365}\right] \\
& +\beta^{\cos } \cos \left[\frac{2 \pi(t+j)}{365}\right]+\varepsilon(t, j), \quad \forall t \in \mathcal{T}, \forall j \in \mathcal{J}
\end{aligned}
$$

Consequently:

$$
\begin{aligned}
\boldsymbol{x}_{t, j} & \left.=\left[\begin{array}{ll}
1 & \sin \left[\frac{2 \pi(t+j)}{365}\right]
\end{array}\right] \cos \left[\frac{2 \pi(t+j)}{365}\right]\right]^{T} \\
\boldsymbol{\beta} & =\left[\begin{array}{lll}
\mu & \beta^{\sin } & \beta^{\cos }
\end{array}\right]^{T}
\end{aligned}
$$

For simplification, the discount rate $r=0$. This entails in $J_{t, i}$ being equal to the swap's $i$ duration in days and arbitrage-free prices the the average of elementary prices defined on its delivery period.

\section{1}

\section{Arbitrage Level Analysis}

Our estimation procedure's first step consisted of calculating the arbitrage-free swap prices $\left(Y_{t, i}\right)$ and filtering contracts with arbitrage levels higher than a pre-defined threshold. To give a sensibility on the arbitrage values observed on both power markets, Fig. 5.1 and 5.2 show the histogram of $\zeta_{t, i}$ for all trading dates $t$ and swaps $i$ for NordPool and BBCE, respectively.

The difference between the arbitrage range of the two markets is clear. While the highest arbitrage detected in NordPool was $0.98 € / \mathrm{MWh}$, in BBCE, the maximum value was $21.6 \mathrm{R} \$ / \mathrm{MWh}(\approx 3.4 € / \mathrm{MWh}$ on the current quote). This is a direct consequence of the market's robustness since the Nordic electricity market has more liquidity, and the Brazilian is not yet consolidated. 


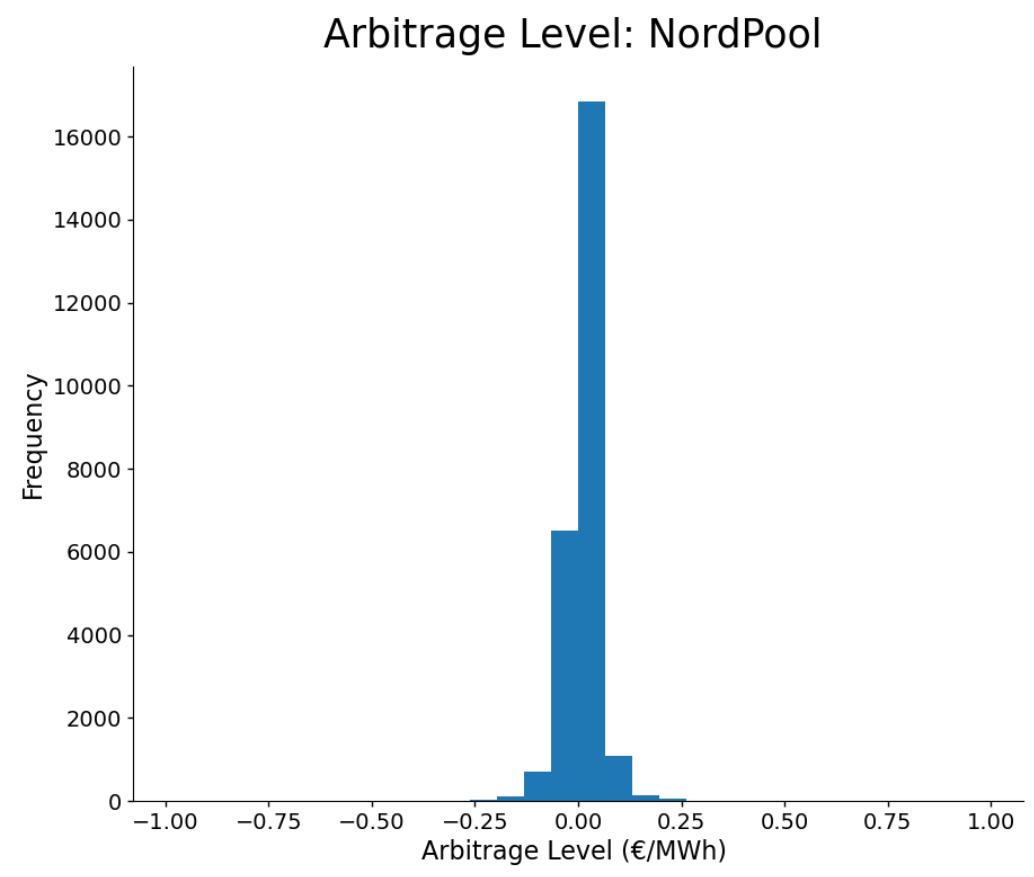

Figure 5.1: Histogram of arbitrage levels in the Nordic power market.

\section{Arbitrage Level: BBCE}

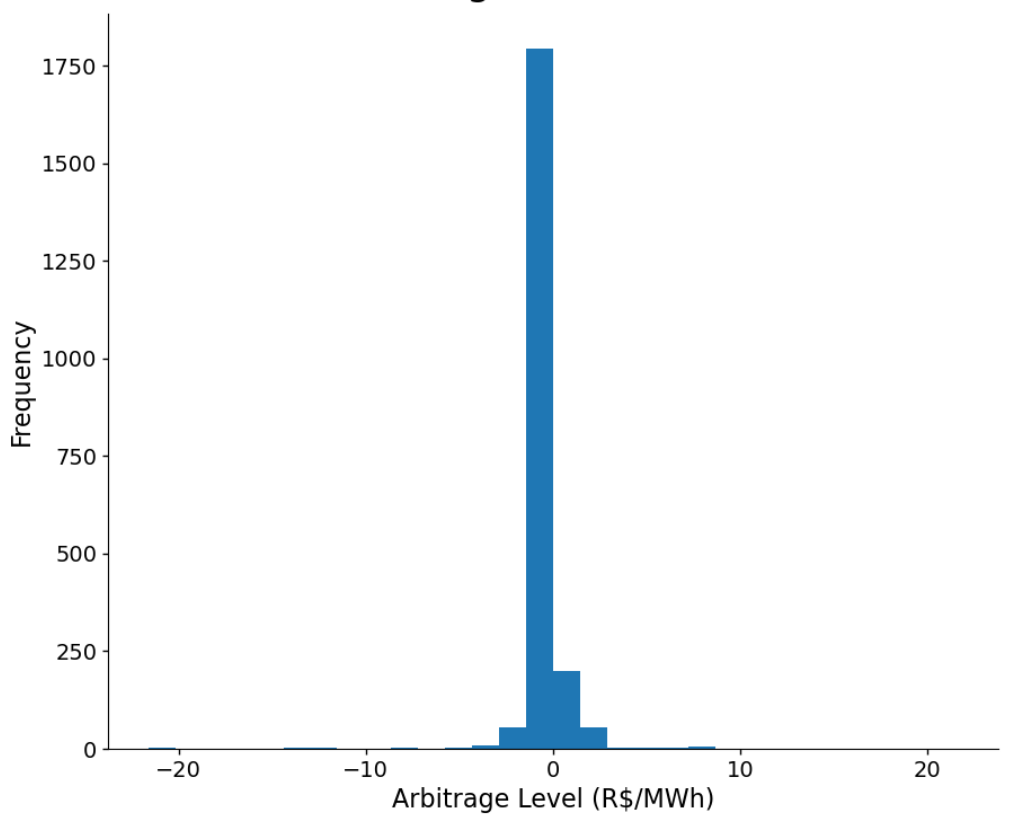

Figure 5.2: Histogram of arbitrage levels in the Brazilian power market.

One interesting and somehow obvious statement is that arbitrage levels different from zero are only observed on completely overlapped contracts. To illustrate, Fig. 5.3 shows the composition between the quarterly and three monthly swaps on August 1st of 2018, the day with the highest levels of arbitrage for the Brazilian data set. 
Settled Prices: Brazil (01/08/2019)

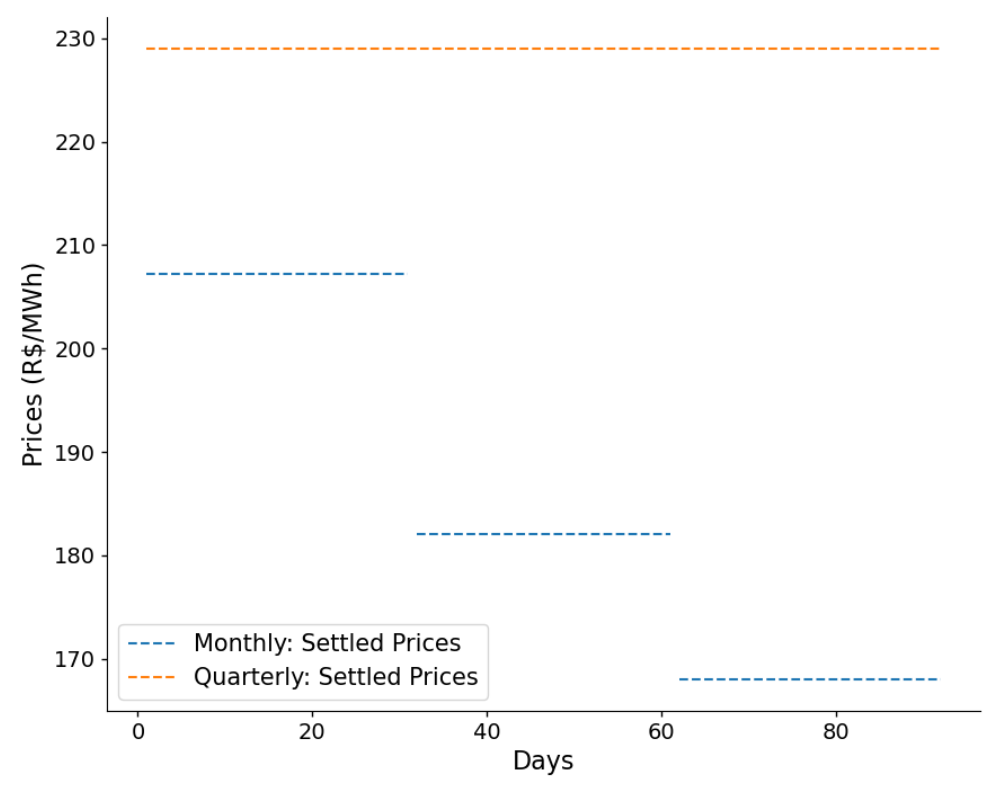

Figure 5.3: Contracts with the highest arbitrage on the Brazilian's forward market.

Approximately each third of a quarterly swap's delivery period is overlapped with a monthly contract, so it is correct to expect the former's price to be essentially an average of the monthly assets. Looking at Fig. 5.3, we can readily conclude that this is unfeasible for the trading day under analysis, as the monthly prices are all lower than the quarterly one. This is reflected in their absolute arbitrage values, which were all around 21.6R $\$ / \mathrm{MWh}$. One could argue why don't just cancel the arbitrage of monthly contracts and keep only the quarterly with a value different from zero and higher than $21.6 \mathrm{R} \$$. This impossibility is a consequence of the algorithm presented in Chapter 4.1, which aims to minimize the maximum arbitrage level on a date $t$ and, as a solution, have a not null arbitrage associated with all the overlapped swaps. The resulting arbitrage-free prices are presented in Fig. 5.4. 
Settled Prices: Brazil (01/08/2019)

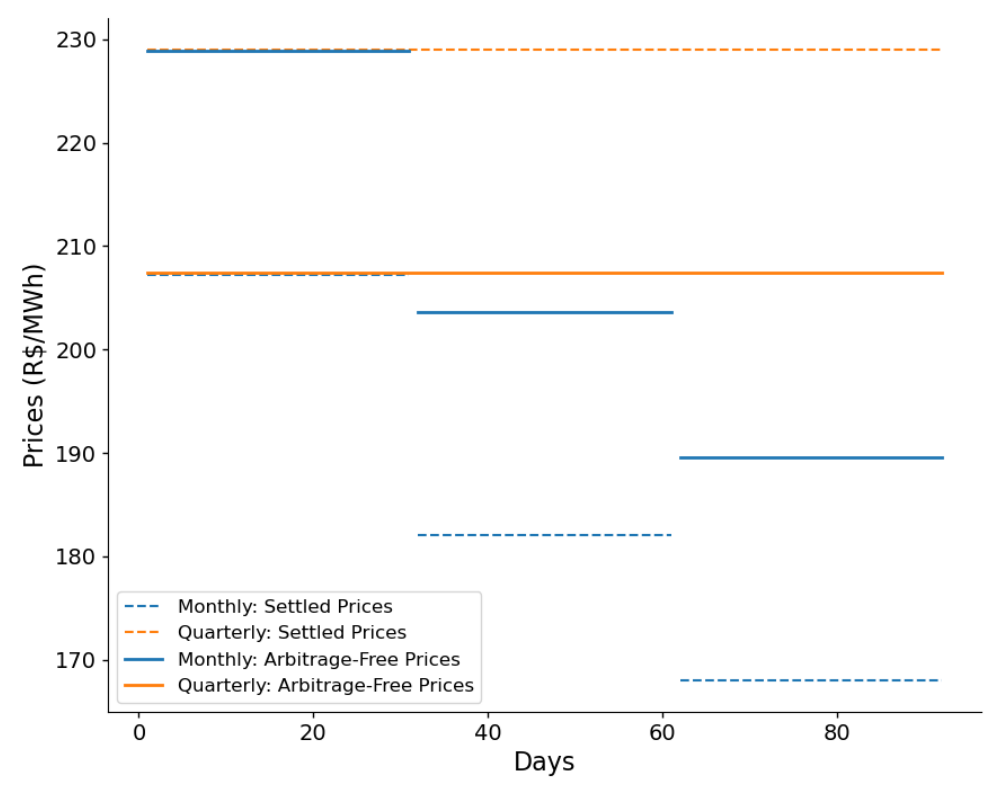

Figure 5.4: Contracts with the highest arbitrage on the Brazilian's forward market and their arbitrage-free prices.

As mentioned before, missing data is a reality when analyzing the Brazilian's future market. Another observed arrangement between overlapped contracts is similar to those presented in Fig. 5.3 and 5.4, but with one of the monthly swaps being unavailable. If it occurs, the elementary forward prices that would compose the missing asset could be determined to make the others' arbitrage equal to zero. This is a limitation of our outlier detection procedure, as abnormal prices can be identified only if their delivery period is wholly overlapped with other settlements.

Finally, the arbitrage threshold acceptable for the Nordic market was $1 € /$ MWh, which means all negotiations remained on the next estimation steps. At first, we've considered an arbitrage limit of $1 \mathrm{R} \$ / \mathrm{MWh}$ to filter the Brazilian data set. However, this would exclude $11.7 \%$ of the available prices. Thus, we've increased this threshold to $2 \mathrm{R} \$ / \mathrm{MWh}$, and then only $3.7 \%$ of traded swaps were disregarded.

\section{2}

\section{Elementary Contracts Estimation}

After calculating the sets of arbitrage-free prices, we proceed to the elementary forward estimation. First, the coefficients $\boldsymbol{\beta}$ were obtained by minimizing the adjustment term in (4-8). We used a trigonometric function for modeling the seasonality on elementary forward. In contrast, using dummy 
variables accounts mostly for shifts in price levels between months [18], and this unnatural non-smooth variation is reflected on the elementary forward curve.

Both Brazilian and Nordic power sectors have hydro-dominant power systems. Therefore, their power prices are lower when higher inflow levels are observed, which occurs in the summer due to rainfall and melting seasons, respectively. Because of their geographic localization, reduced prices are detected in opposite months of the year. Fig. 5.5 shows the normalized values of the estimated trigonometric function for the markets under analysis.

\section{Seasonality: Elementary Forward Contracts}

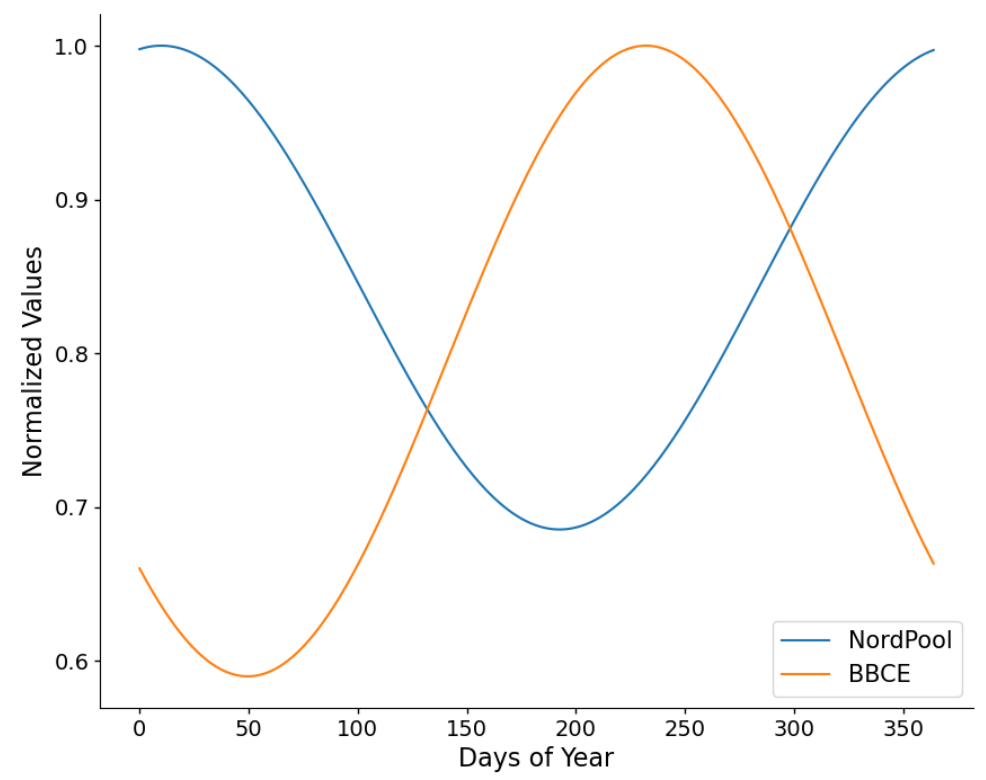

Figure 5.5: Normalized seasonality functions.

With the vector $\boldsymbol{\beta}$ fixed, we followed to the maximum smoothness criterion on the residuals. In the last stage, the problem (4-9)-(4-10) was solved for the Nordic data set, while the addition of time-dependency was made to address the scarcity of data from the Brazilian's forward market. As the two objectives in (4-11) might be on different scales, depending on the values of $\lambda_{1}$ and $\lambda_{2}$, the scaled objective would always be prioritized if its weight is not small enough. Therefore, before solving the bi-objective optimization, we've calculated the objective function values of two optimization problems that maximize the smoothness in time and maturity, separately. Then $\lambda_{1}$ and $\lambda_{2}$ are normalized by the total curvature obtained in maturity and time, respectively. Now, any pair of weighting parameters correctly reflects the specified priority relationship. 
Regardless of whether the second smoothing objective is included, constraints (4-10) and (4-12) guarantees that the elementary forward contracts perfectly recover the available arbitrage-free prices. Nevertheless, to exemplify the bi-objective formulation's impact on the estimation of missing prices, we analyze the trading date May 2nd of 2018, from the BBCE forward market. On this day, only the M+1 swap, which is equivalent to the product JUN/2018, was negotiated. If the maximum smoothness criterion was applied just on the maturity dimension, the residuals $\varepsilon_{t, j}$ for $j>h_{t, i}+\Delta T_{t, i}$, with $i$ being the settled contract JUN/2018, would be determined only by the curvature minimization in $j$, since there are no prices to base them on. The optimal solution would then be maintaining the final slope of the residuals correspondent to the $\mathrm{M}+1$ price, as shown in Fig. 5.6. Analogous arguments can be made on other trading days of the Brazilian set, when alternative prices are unavailable due to the market's low liquidity.

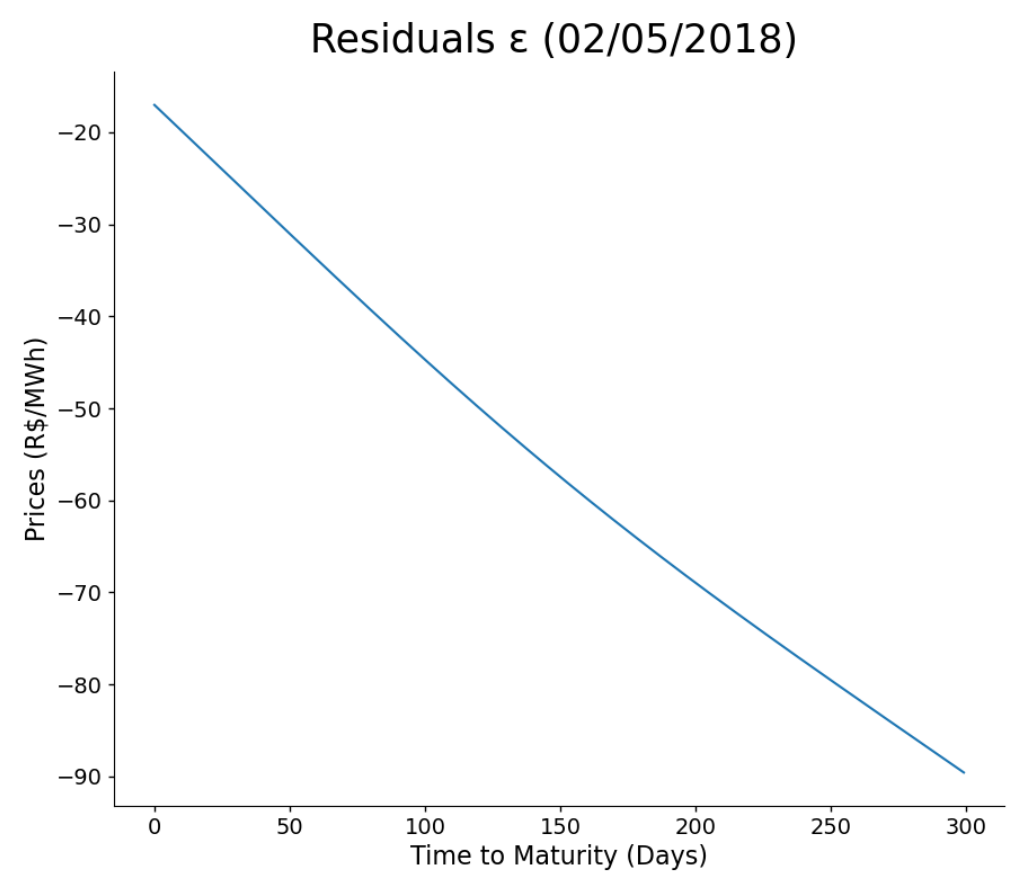

Figure 5.6: Estimated residuals on March 2nd 2018 without maximum smoothness criterion in time.

In contrast, the bi-objective smoothing process doesn't allow the residuals to differ too much from those on days close to each other. Hence, even though only the $\mathrm{M}+1$ price is available on May 2nd of 2018, the remaining maturities will be influenced by prices settled on other trading days. This impact is shown in Fig. 5.7. 


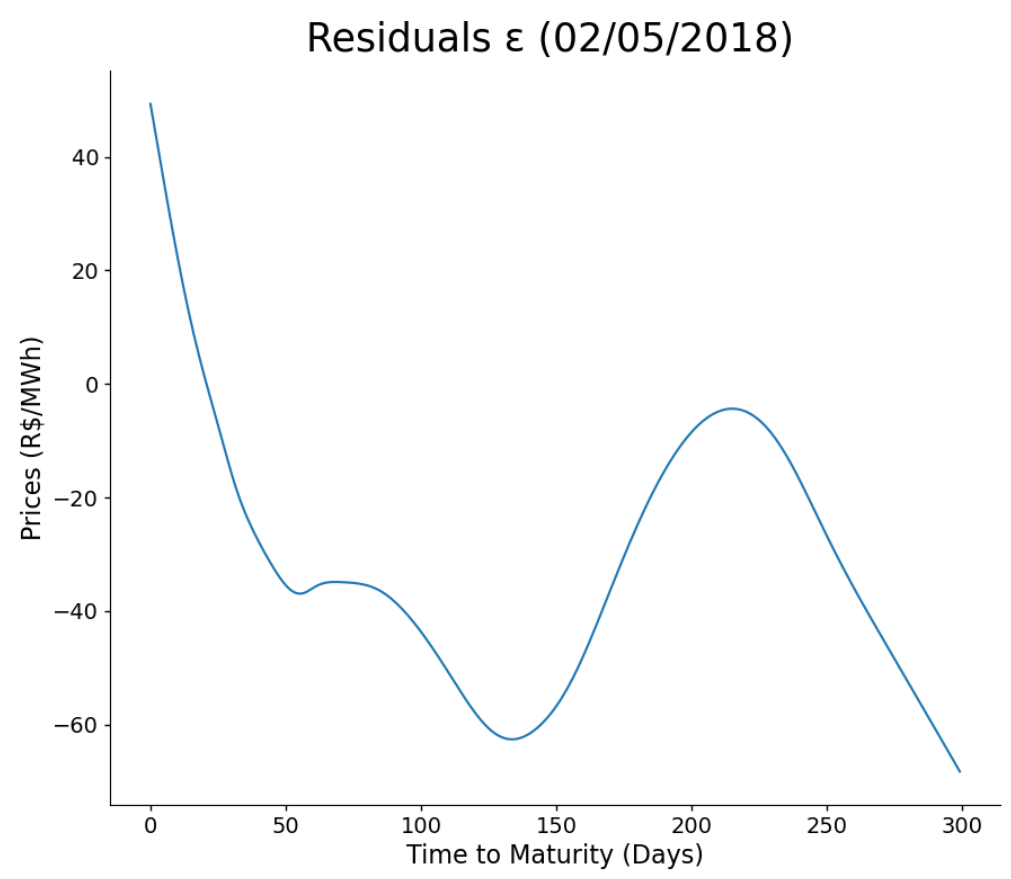

Figure 5.7: Estimated residuals on March 2nd 2018 with maximum smoothness criterion in time.

To understand how this reflects in the time series built by calculating the missing swaps with equation (4-5), we show in Fig. 5.8 - Fig. 5.15 the resulted prices for all time series from BBCE, with and without the smoothing in time dimension. It is notorious how this approach is essential to reduce the noise level of the estimated missing prices and promotes a more reasonable result. 
$M+1$

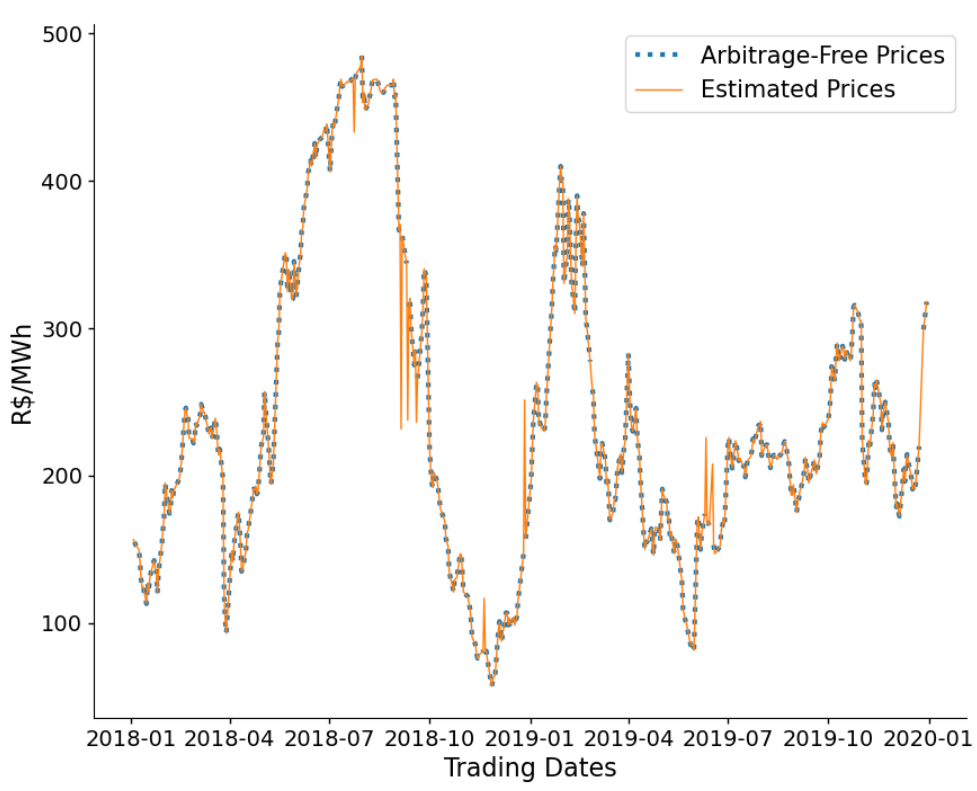

5.8(a): Smoothing only in maturity.

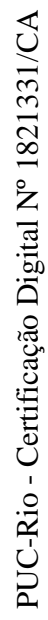

$M+1$

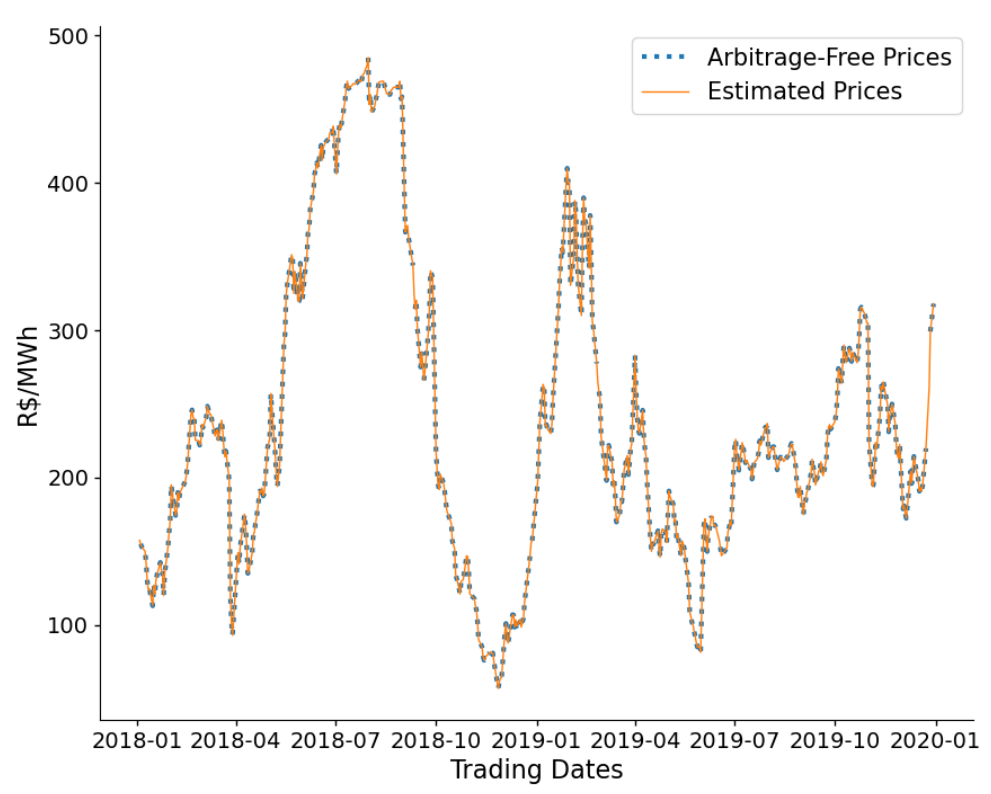

5.8(b): Smoothing in time and maturity.

Figure 5.8: $\mathrm{M}+1$ arbitrage-free swaps from BBCE. 
$M+2$

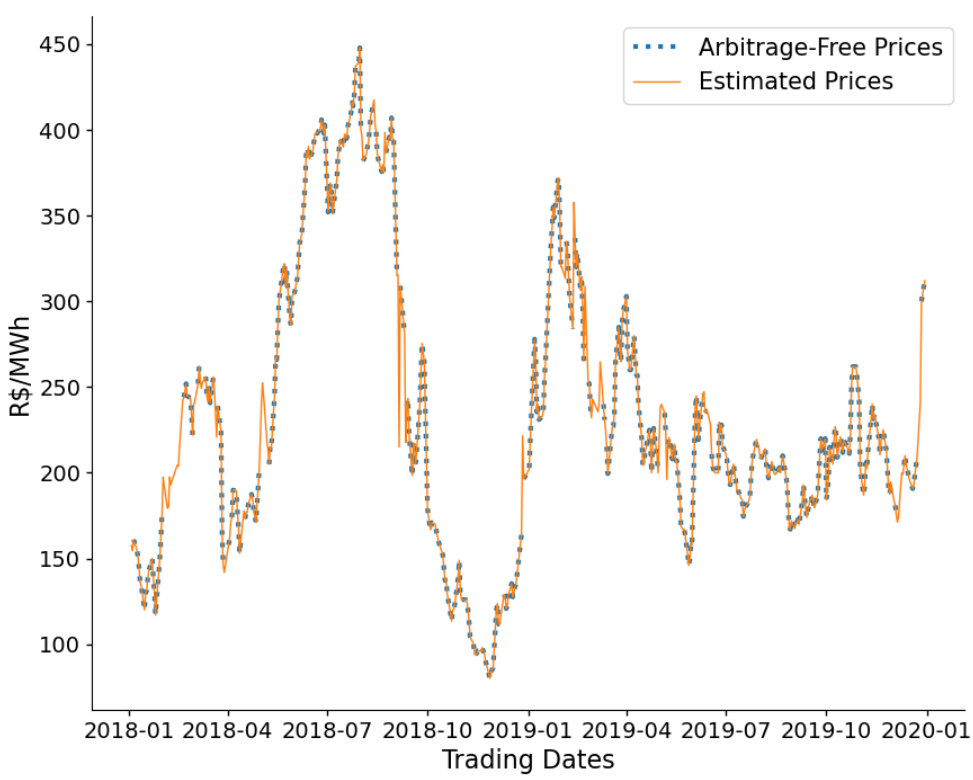

5.9(a): Smoothing only in maturity.

$M+2$

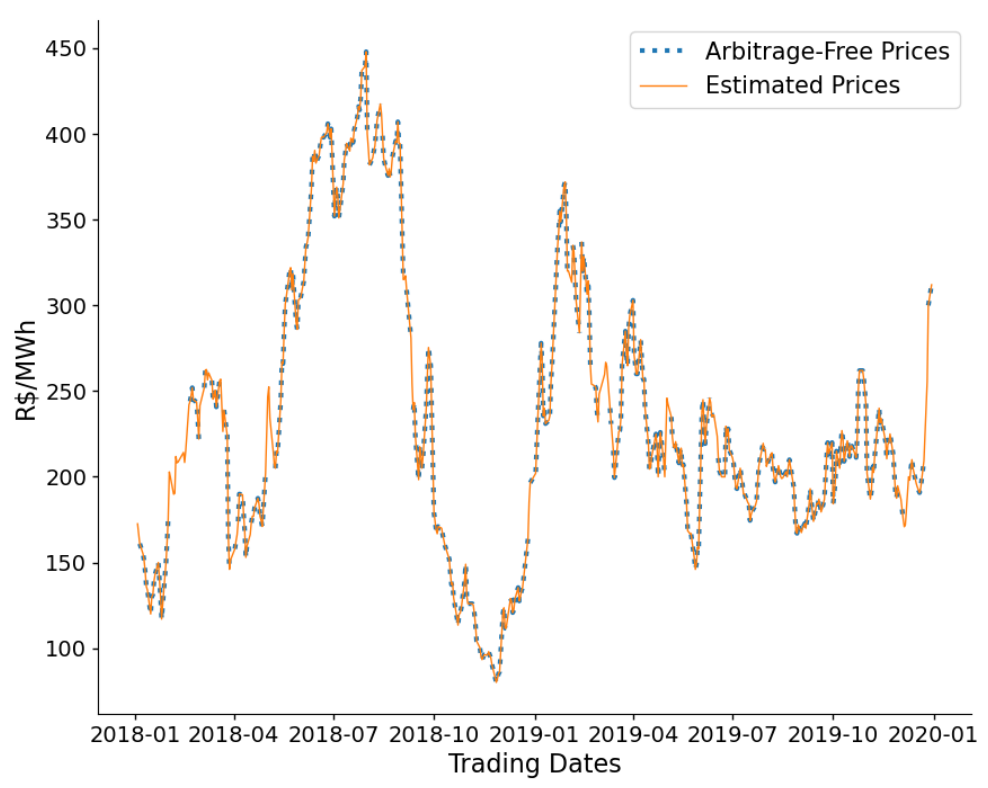

5.9(b): Smoothing in time and maturity.

Figure 5.9: $\mathrm{M}+2$ arbitrage-free swaps from BBCE. 
$M+3$

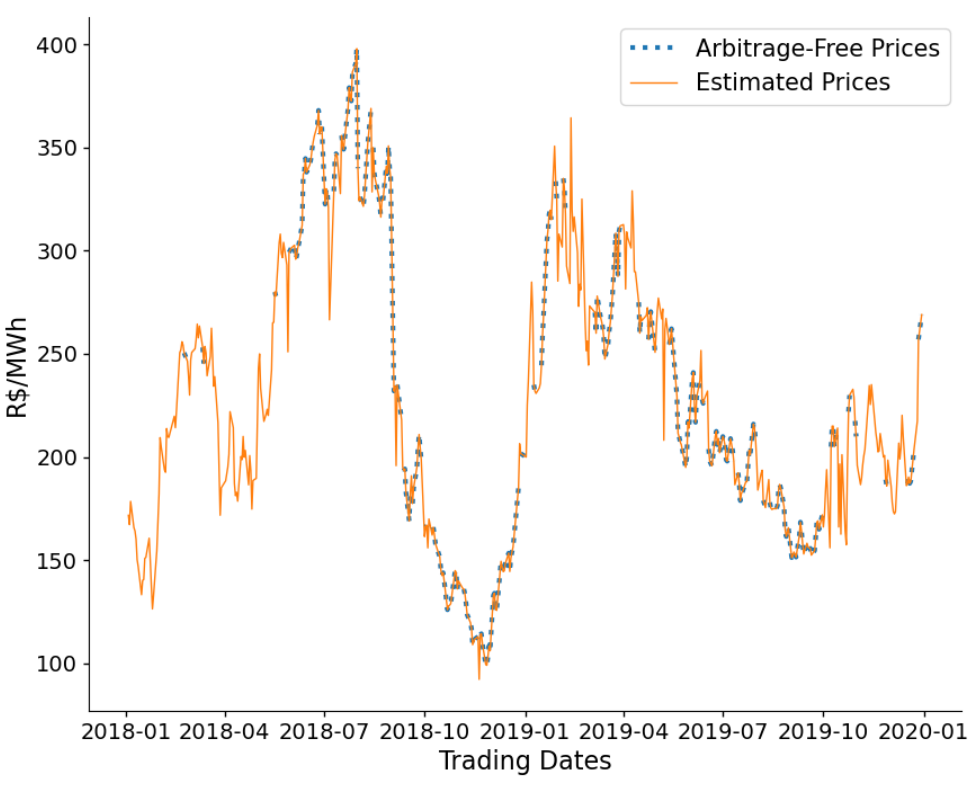

5.10(a): Smoothing only in maturity.

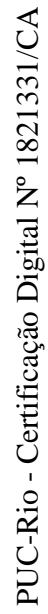

$M+3$

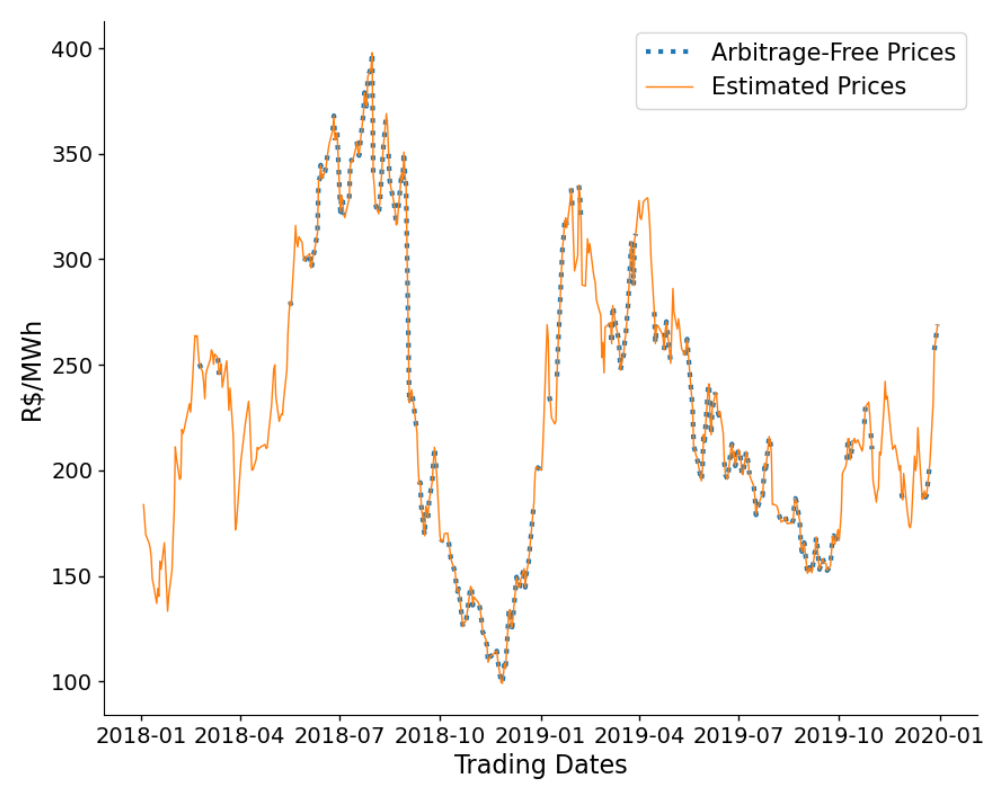

5.10(b): Smoothing in time and maturity.

Figure 5.10: $\mathrm{M}+3$ arbitrage-free swaps from BBCE. 


\section{$M+4$}

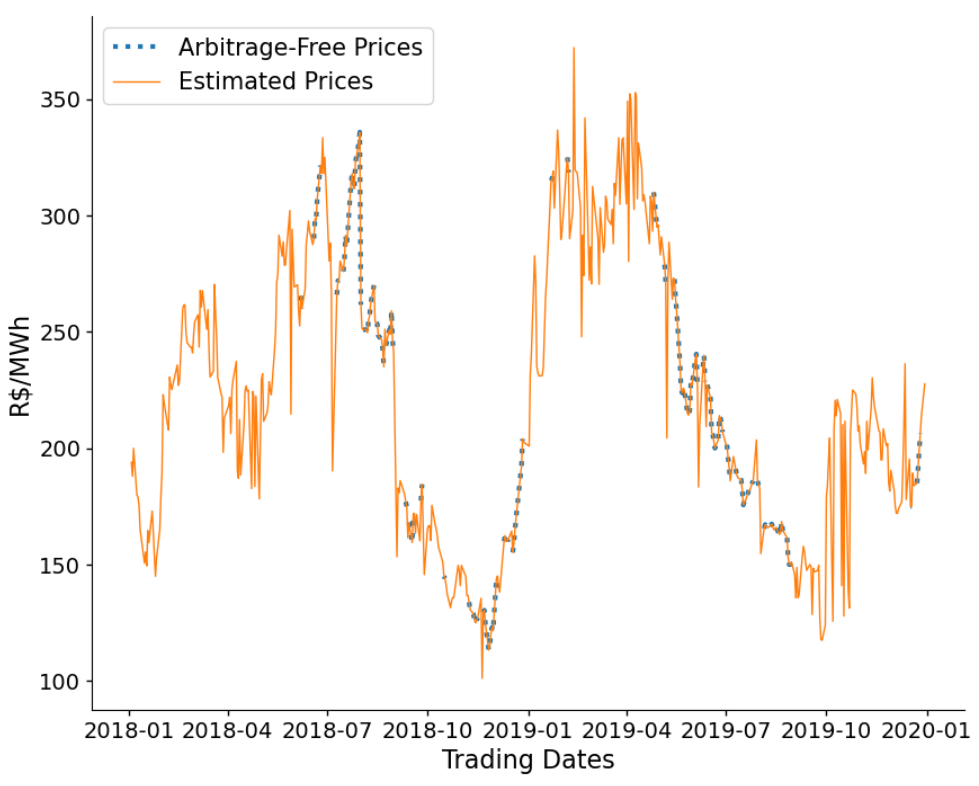

5.11(a): Smoothing only in maturity.

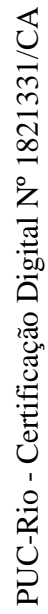

\section{$M+4$}

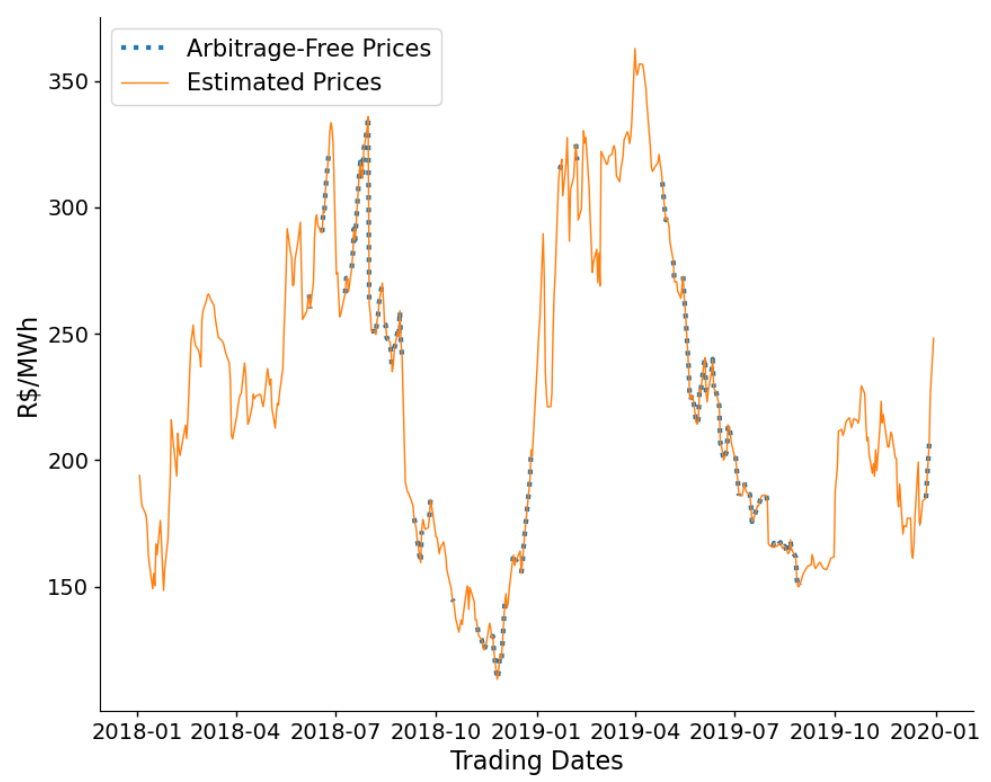

5.11(b): Smoothing in time and maturity.

Figure 5.11: $\mathrm{M}+4$ arbitrage-free swaps from BBCE. 
$M+5$

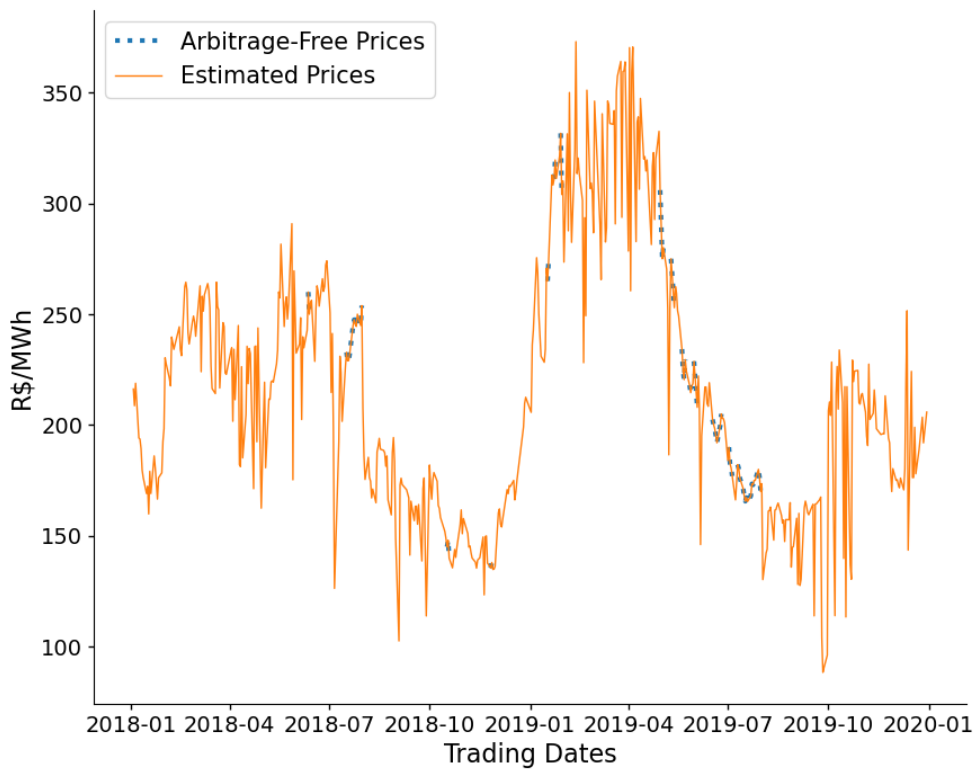

5.12(a): Smoothing only in maturity.

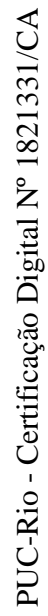

\section{$M+5$}

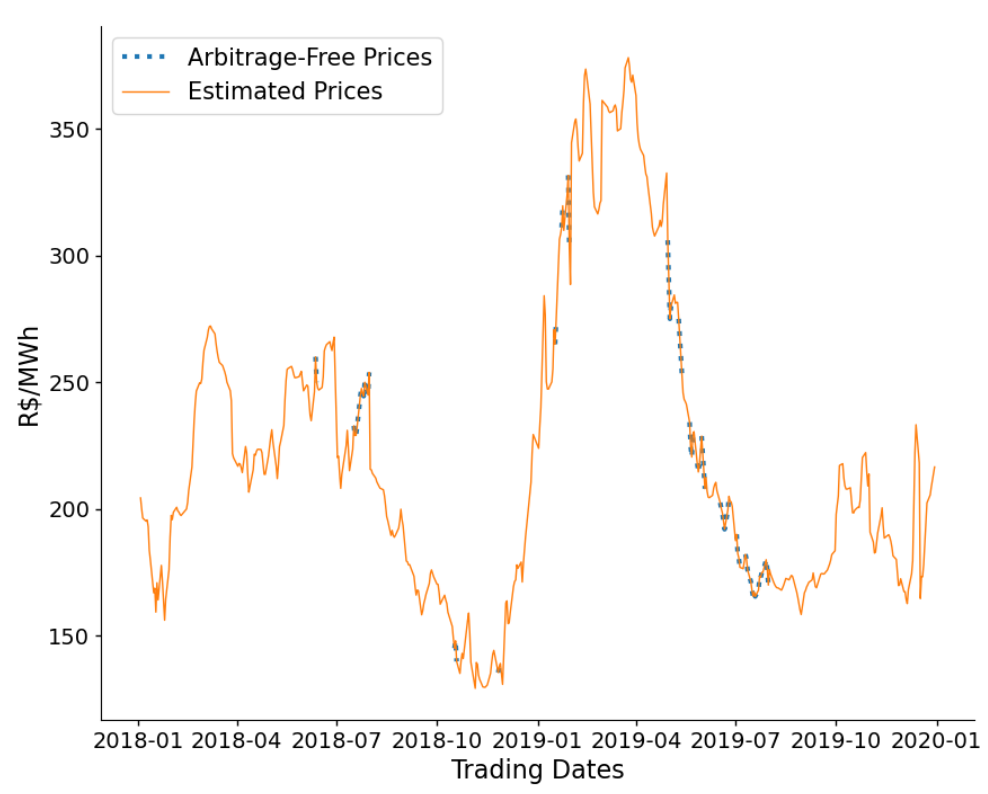

5.12(b): Smoothing in time and maturity.

Figure 5.12: $\mathrm{M}+5$ arbitrage-free swaps from BBCE. 
$M+6$

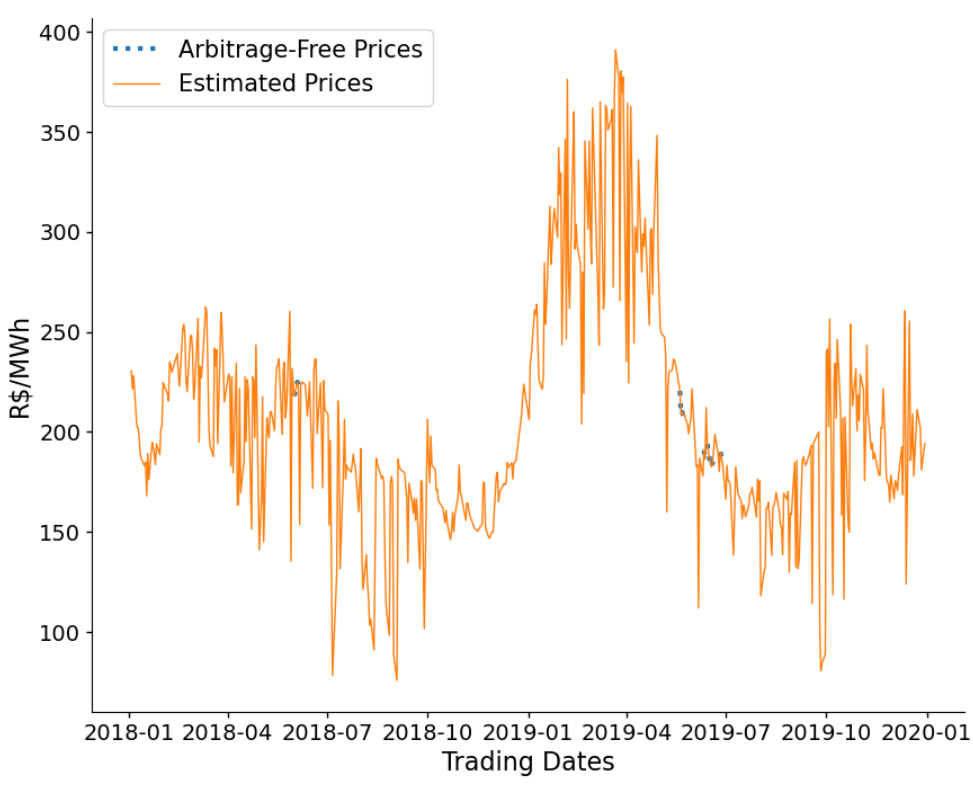

5.13(a): Smoothing only in maturity.

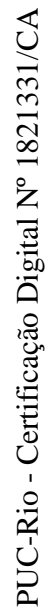

\section{$M+6$}

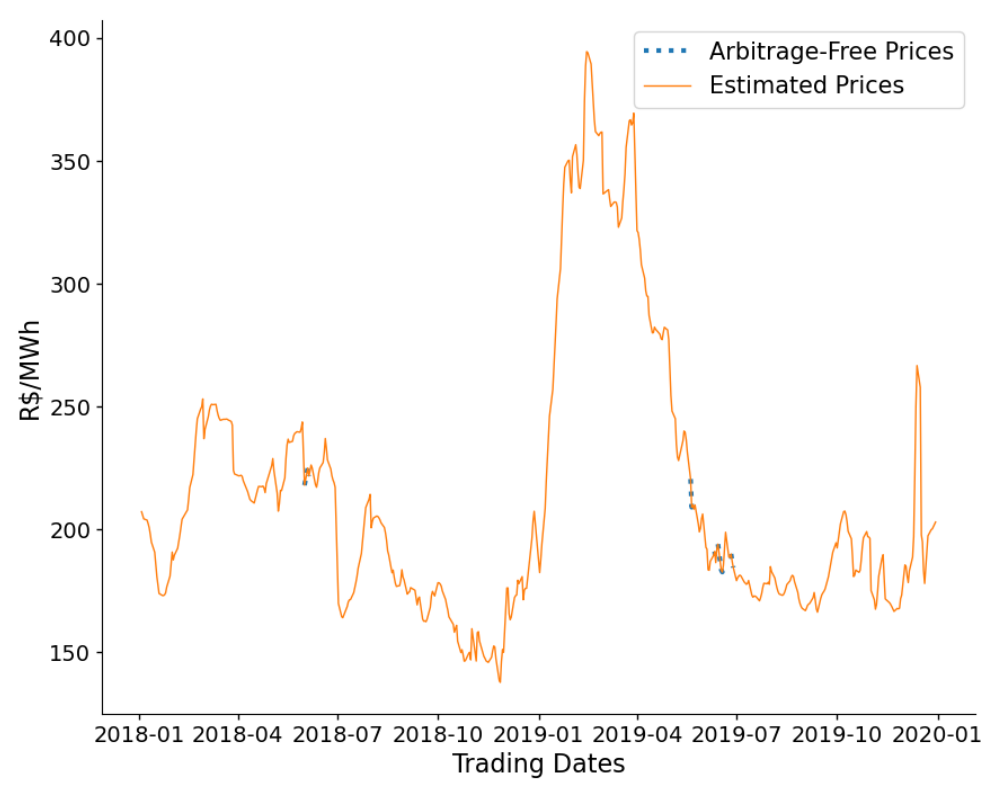

5.13(b): Smoothing in time and maturity.

Figure 5.13: $\mathrm{M}+6$ arbitrage-free swaps from BBCE. 


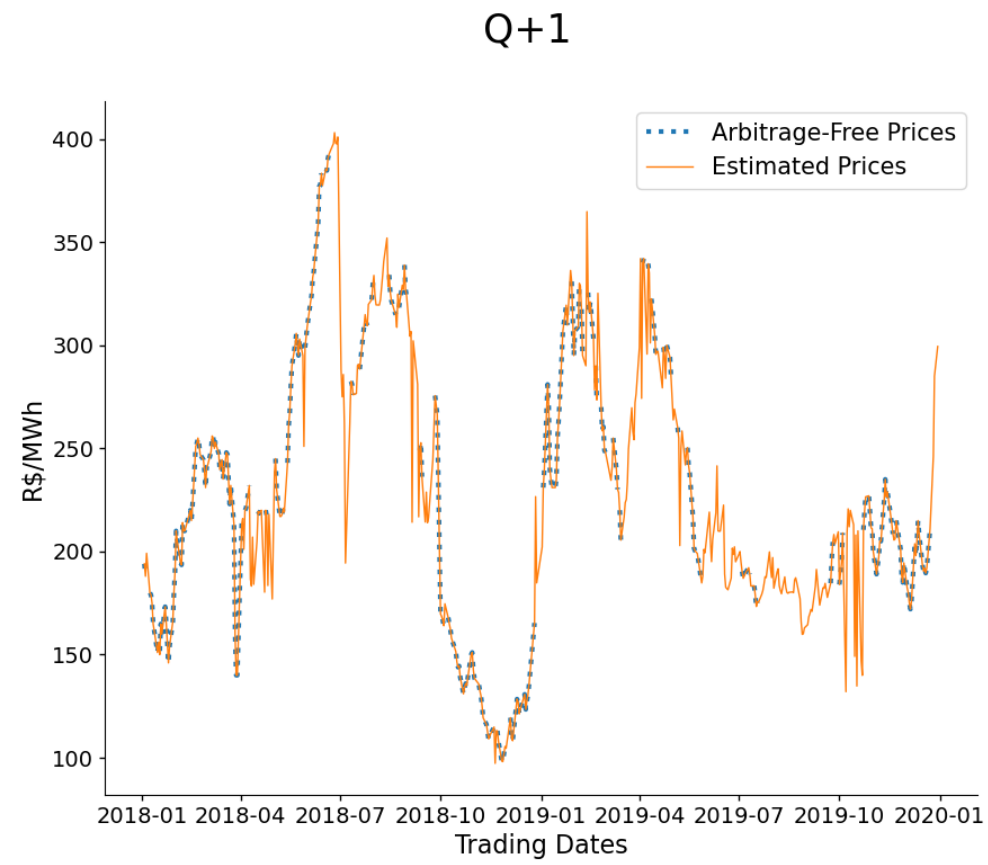

5.14(a): Smoothing only in maturity.

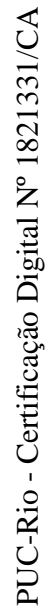

$$
\mathrm{Q}+1
$$

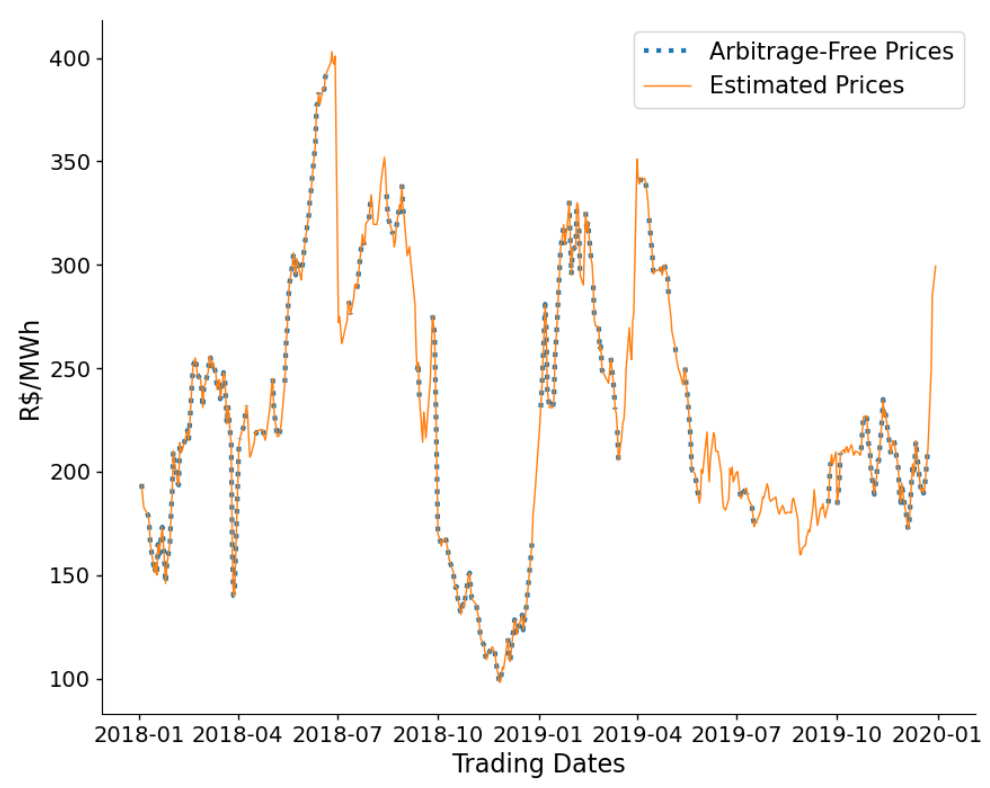

5.14(b): Smoothing in time and maturity.

Figure 5.14: Q+1 arbitrage-free swaps from BBCE. 


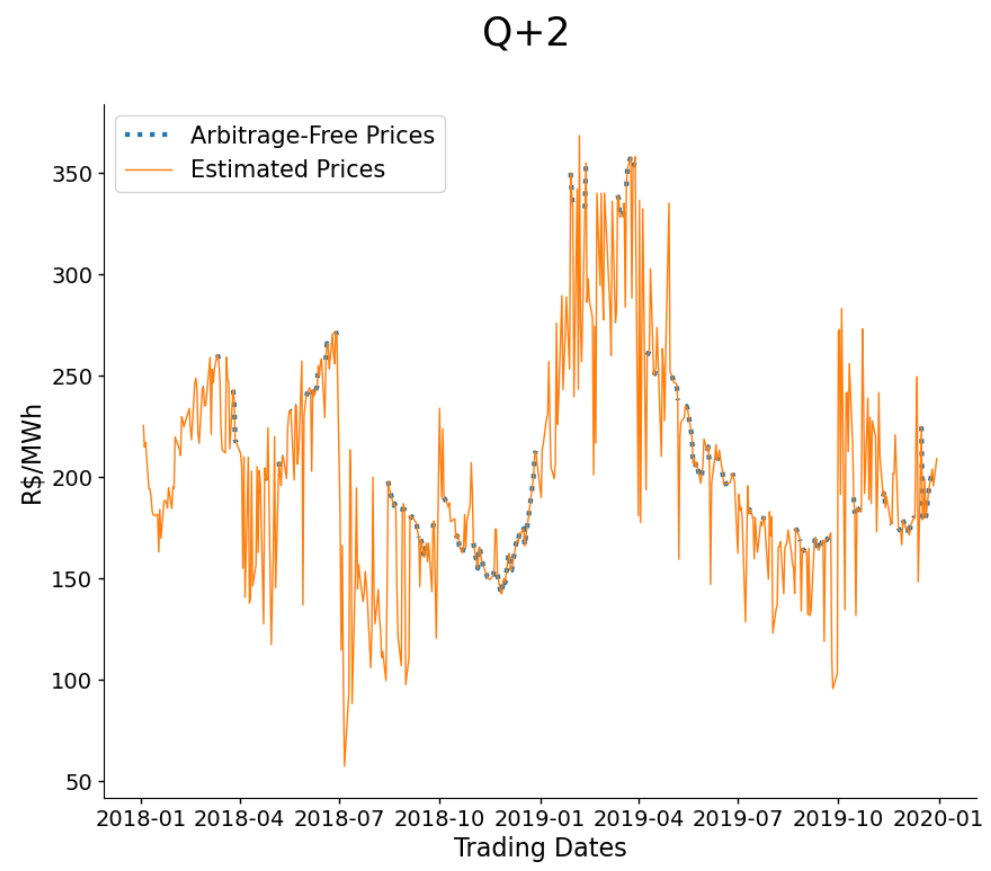

5.15(a): Smoothing only in maturity.

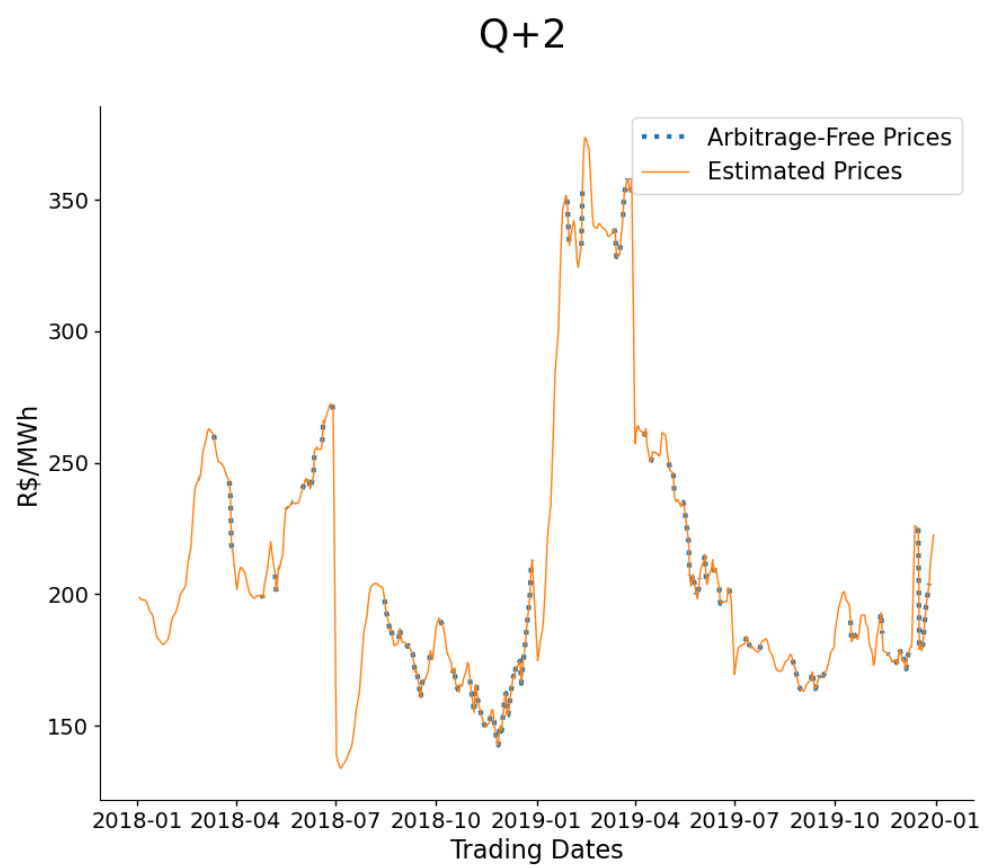

5.15(b): Smoothing in time and maturity.

Figure 5.15: Q+2 arbitrage-free swaps from BBCE.

Finally, we illustrate in Fig. 5.16 and Fig. 5.17 the continuous forward curves of a specific trading date from NordPool and BBCE, respectively, and the arbitrage-free prices for both days. 
Forward Curve: NordPool (03/08/2018)

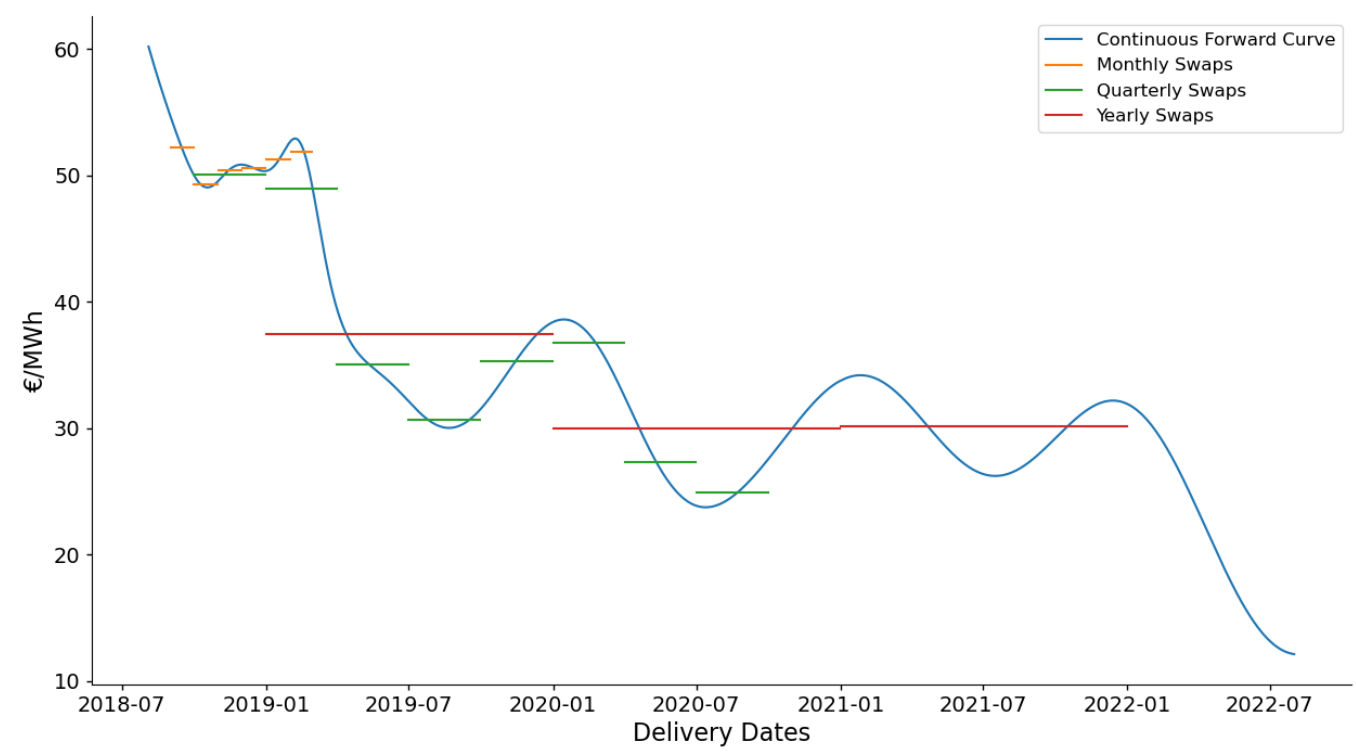

Figure 5.16: Continuous forward curve and arbitrage-free swaps from NordPool.

Forward Curve: BBCE (23/05/2019)

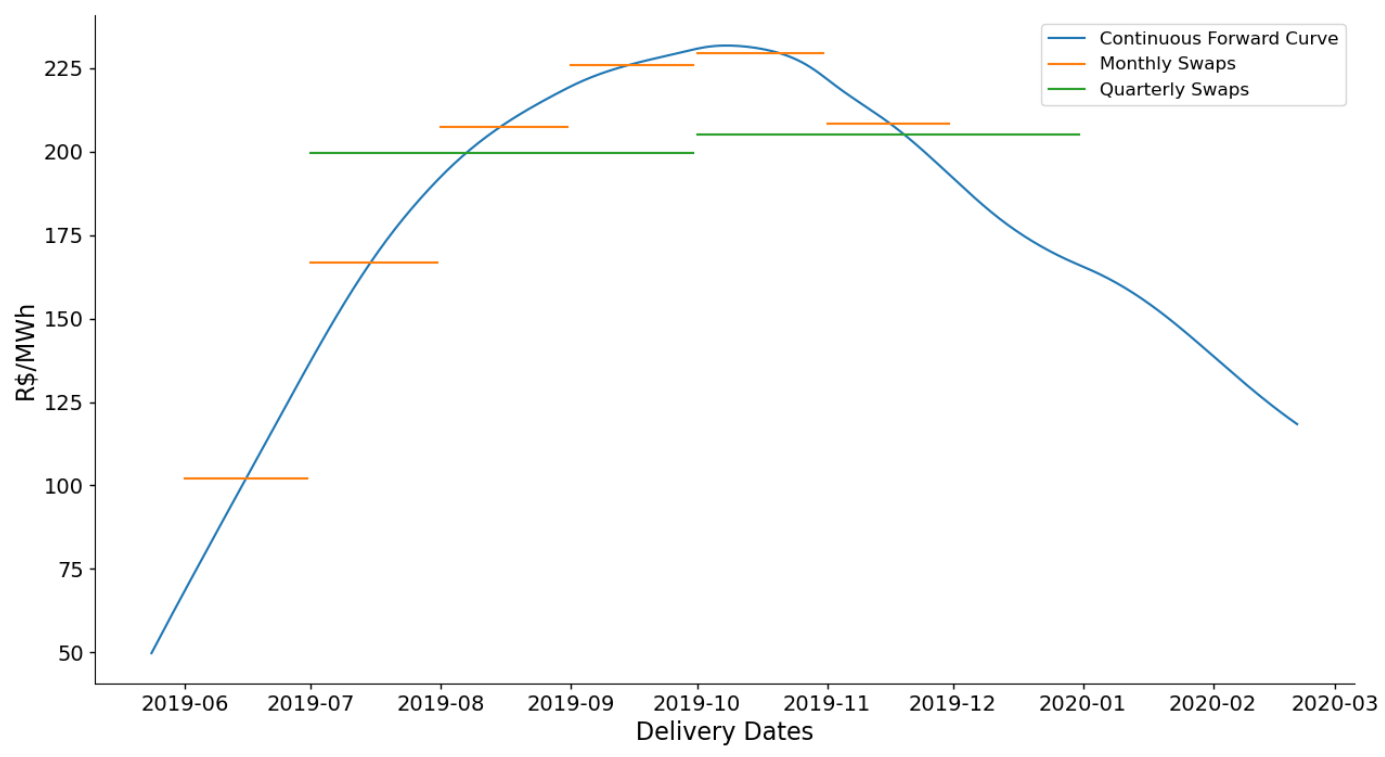

Figure 5.17: Continuous forward curve and arbitrage-free swaps from BBCE. 


\section{3 \\ Dimensionality Reduction}

Our estimation procedure results in two data sets: the first is composed of the elementary forward prices $\left(f_{t, j}\right)$ and the second by their respective residuals $\left(\varepsilon_{t, j}\right)$. In the context of this study, PCA decomposes the matrix of residuals in two separate sets: one of time-indexed series, called components, and other of their factor loadings, which varies with maturity $j$ and represents the weight of each component on $\varepsilon_{t, j}$. To evaluate this approach, we analyze the following aspects of the PCA outcomes:

1. The number of components necessary to explain a high percentage of the variance of the residuals. As PCA's objective is dimensionality reduction, its use is only reasonable if the number of components is significantly lower than the number of estimated maturities.

2. Interpretability and generalization of factor loadings. It's important to understand how different parts of the curve are affected by each component. The factor loadings shapes are usually a subject under study, as in [2] and [10]. The factor loading's generalization will be discussed later on in this chapter.

An issue regarding PCA's performance on the residuals matrix, or even on the elementary forward prices, if that was the case, is until which maturity we should consider when obtaining the components. To clarify, assume the NordPool settlements, with $\mathrm{Y}+3$ being the swaps with the biggest time to maturity. If a $\mathrm{Y}+3$ is traded on January 1st, for instance, the highest maturity $j$ that would have its residuals based on some price would be approximately $4 * 365=1460$ (the first $3 * 365=1095$ correspond to its time to maturity and the last 365 days to the delivery period). Now, suppose the same product is negotiated on December 31st of the same year. The highest maturity would then reduce to $3 * 365=1095$, since the $\mathrm{Y}+3$ asset's maturity decreased in approximately one year. Visually, this implicates the following behavior on the residuals matrix. 


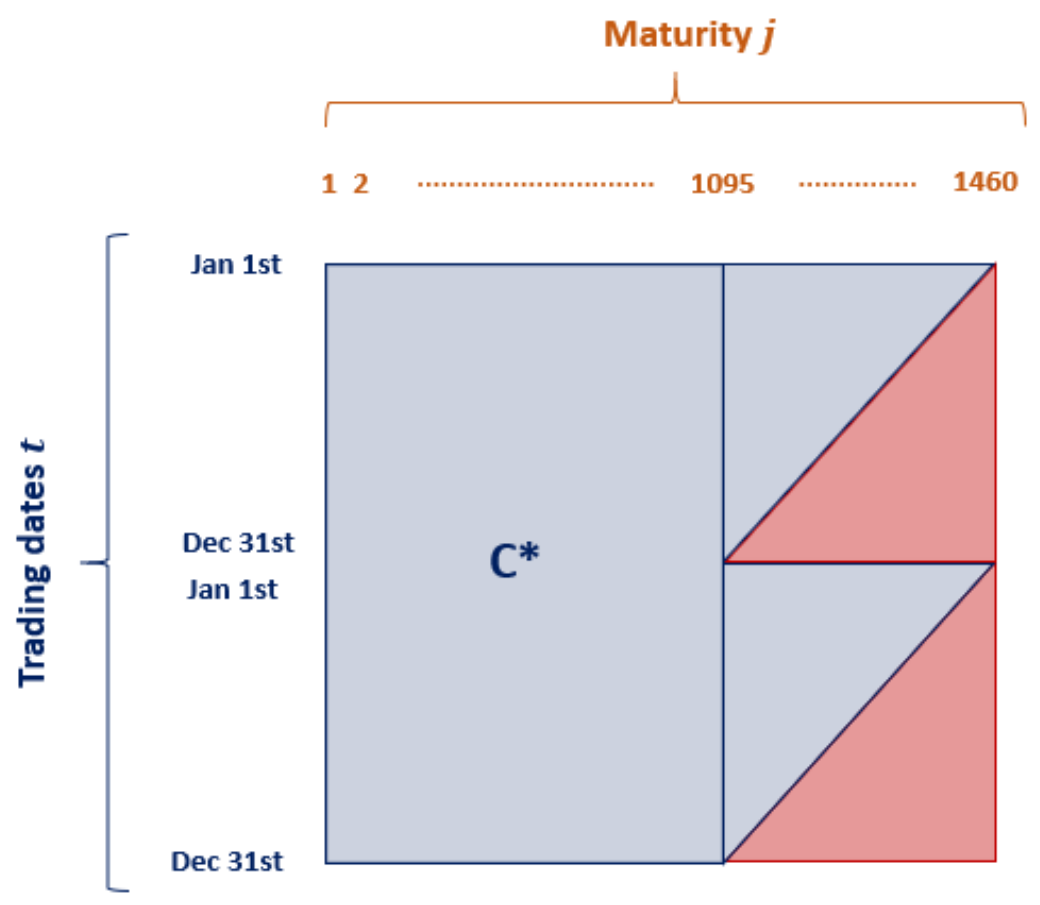

Figure 5.18: Maximum maturity reduction between trading days.

The convex set $\mathcal{C}^{*}$ refers to the maturities, guided by both prices and smoothness, common to all trading dates. It's crucial to emphasize that this shape is an inherent characteristic of the calculated data sets, regardless of what swaps are settled. The only distinction is when the illustrated breaks occur. For example, for the Brazilian assets, because the highest time to maturity and duration is from the $\mathrm{Q}+2$ contracts, the discontinuities arise when the quarter changes.

For the maturities inside the red triangles, their residuals are guided solely by the maximum smoothness criterion. The issues related to the curvature minimization only on the maturity dimension have already been highlighted. But even the incorporation of the time-dependency might not be enough in such cases, since its influences are somehow restricted to trading days close to each other. These conclusions indicate how powerful our framework is to interpolation purposes but limited for extrapolation.

Therefore, PCA was performed on the convex set $\mathcal{C}^{*}$ of the residuals matrices from NordPool and BBCE, so the negative impacts of extrapolation do not interfere with the results. Three components were able to explain $97.4 \%$ (NordPool) and 98.4\% (BBCE) of the variance of the residuals. Fig. 5.19 and 5.20 illustrates the computed factor loadings. 


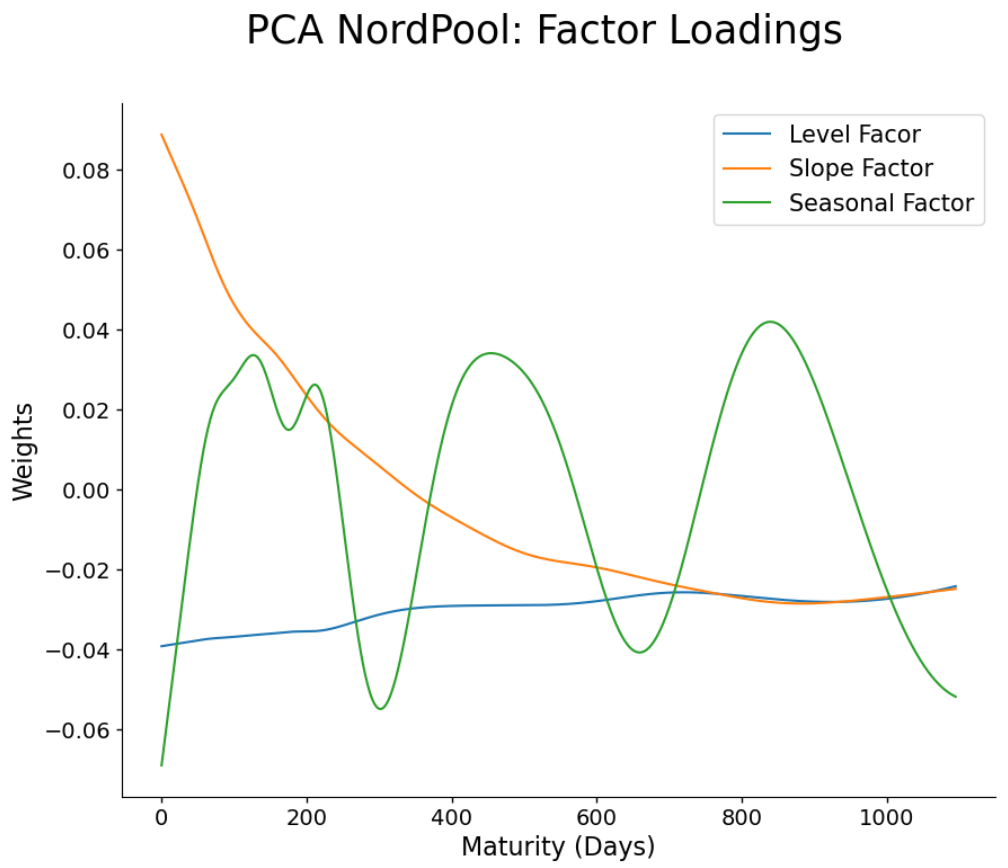

Figure 5.19: First three factor loadings from NordPool residual's data set.

PCA BBCE: Factor Loadings

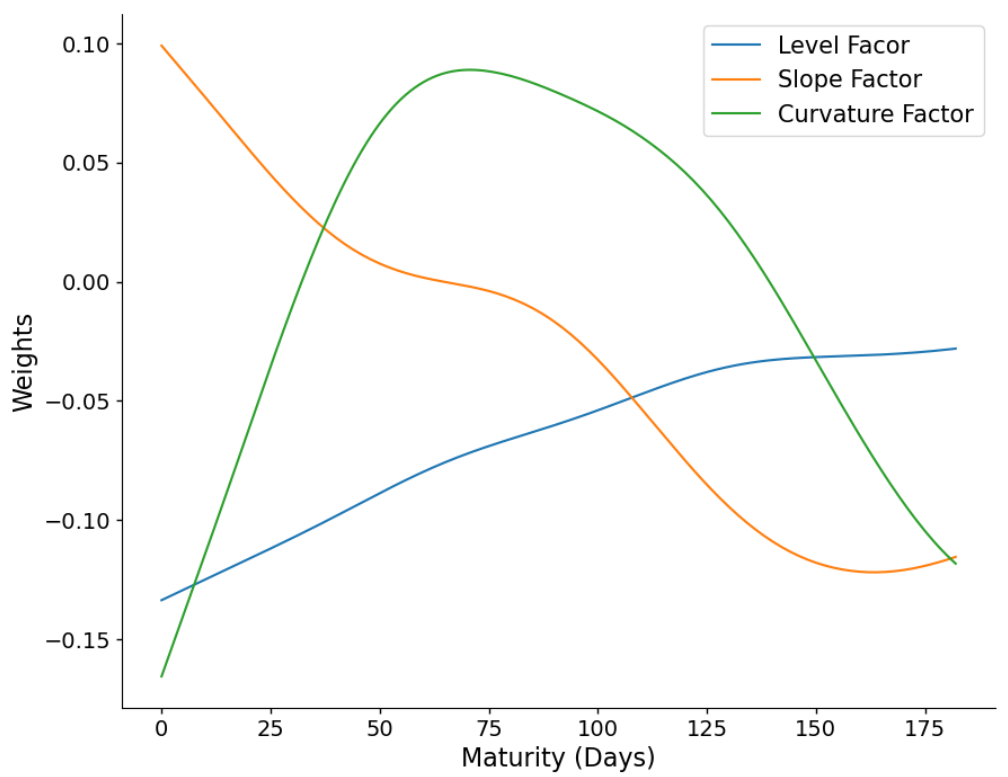

Figure 5.20: First three factor loadings from BBCE residual's data set.

The first two factors found on both sets are well-known in the literature: the level factor represents changes on a constant rate across maturities, while the slope or steepness exhibit opposite signals between the curve's short and long end. Furthermore, the third factor of NordPool still maintains a seasonal 
structure. In contrast, for BBCE, another traditional factor, referred to as curvature, was observed, where the short and long end of the curve have the same signal, yet the middle presents an opposite one.

The percentage of explained variance in our study was above of what was found in other studies regarding electricity forward curve modeling. We believe this is mainly because we perform PCA on the residuals, instead of directly on the elementary forward prices. Fig. 5.21 and 5.22 presents the correspondent extracted components.

\section{PCA NordPool}

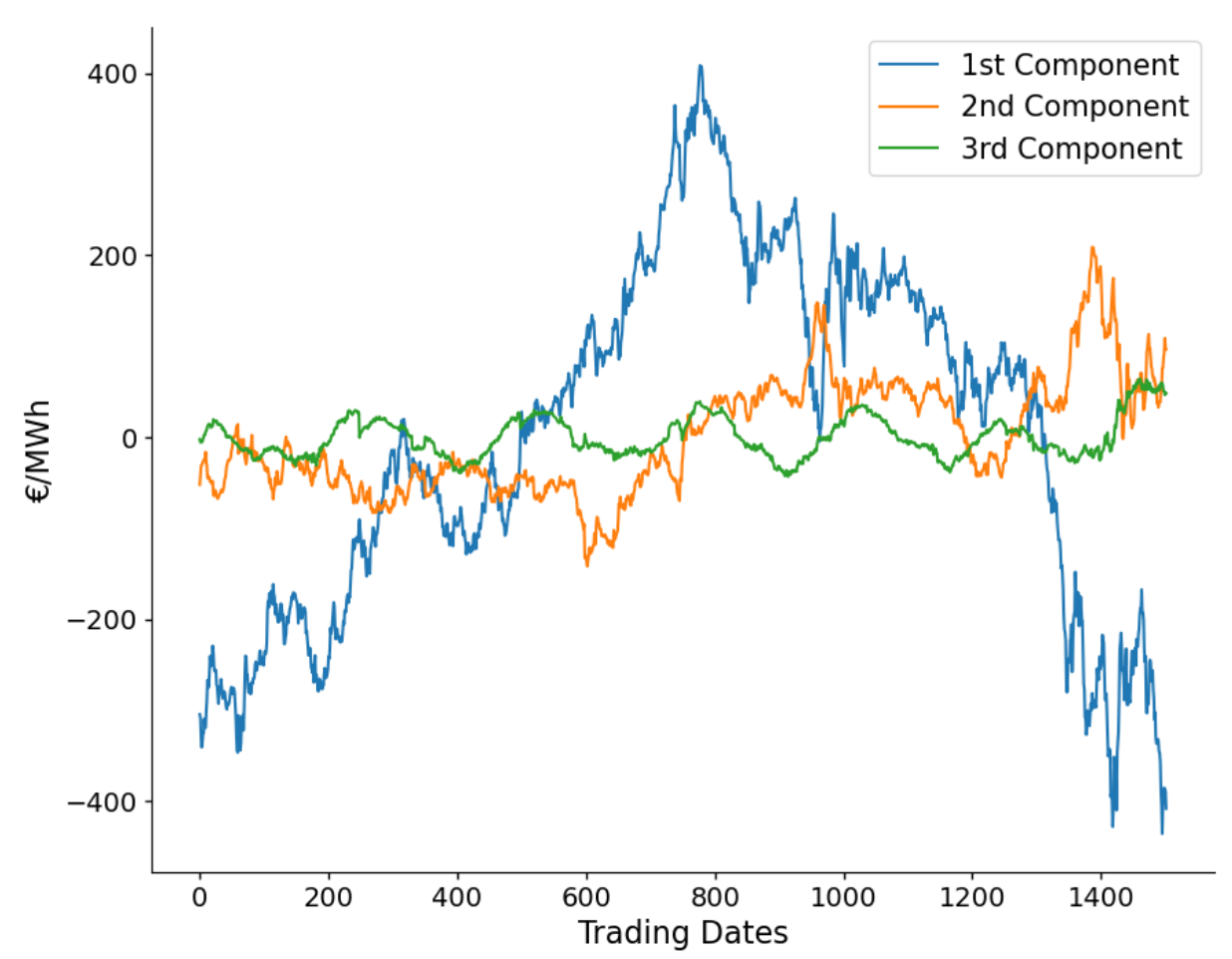

Figure 5.21: Components from the NordPool data set. The ADF-Test was not able to reject the null hypothesis of existing a unit root $(10 \%, 1 \%, 10 \%$ levels of significance, for each component, respectively). 


\section{PCA BBCE}

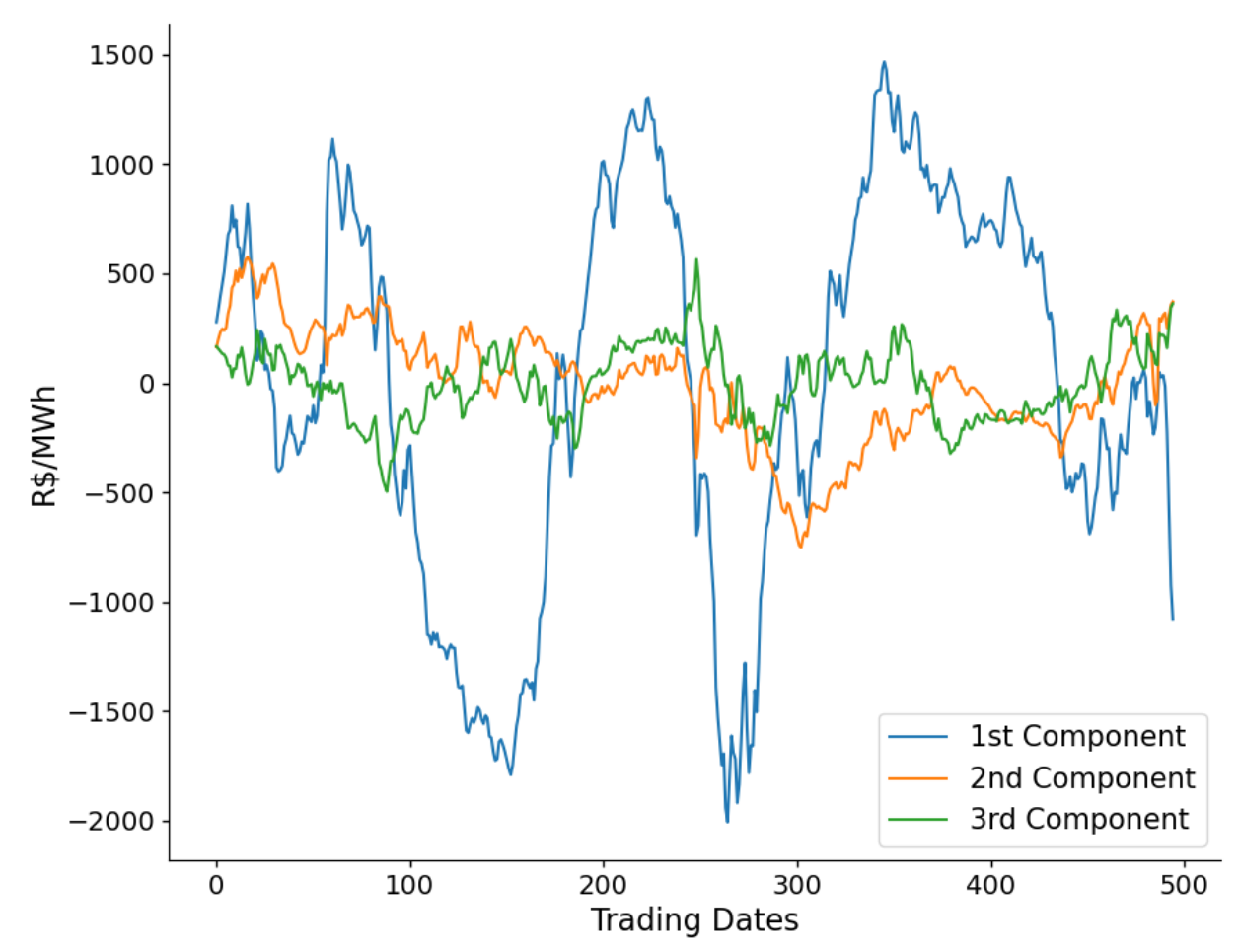

Figure 5.22: Components from the BBCE data set. The ADF-Test was not able to reject the null hypothesis of existing a unit root for the first two components ( $5 \%$ and $1 \%$ levels of significance, respectively). In contrast, the null hypothesis was rejected for the third component.

As the PCA was performed under a truncated data set, the weight's values $w_{j, k}$ are defined just for the maturities $j$ of residuals $\varepsilon_{t, j} \in \mathcal{C}^{*}$. Hence, because we may be interested in extend the use of our structural model for predicting purposes (which will be discussed in Chapter 7), and predicting swap prices composed by higher maturities than those considered is a reality, we must extrapolate the factor loadings. When forecasting, the factor loading's generalization plays an important role. By generalization, we mean that they shouldn't have any remaining structure, and therefore, their parametric representations are more straightforward and stable in some sense. Consequently, the seasonal factor from the NordPool data set was disregarded in this stage. The remaining seasonal behavior should be addressed by updating the structure imposed on the elementary forward contracts. Its presence indicates that probably another periodic pattern has not yet been disclosed by (5-1). Adding more harmonics could assist in this process. Nevertheless, the level and slope factors still explain a total of $96.3 \%$ of residuals variance, 
which is also an impressive result.

We've fitted three parametric representations for the level, slope, and curvature factors, respectively, allowing us to obtain the weight's values for any desired maturity.

$$
\begin{aligned}
& \text { Level }: w(j)=a+b j \\
& \text { Slope : } w(j)=a((1-c) \exp (-b j)+c) \\
& \text { Curvature }: w(j)=a((1-c) \exp (-b j)+c) \\
& \qquad \begin{array}{c} 
\\
+
\end{array}
\end{aligned}
$$

The level is easily described by a first-order polynomial equation, while the slope has an exponential decay to account for the empirical evidence of volatility falling sharply with maturity in the short end and stabilizing in the long end. This attribute, as well as its parametric representation, was discussed in [2]. Finally, the curvature factor is a sum of the previous exponential decay and a second-order polynomial equation to address the concavity. Fig. 5.23 and 5.24 illustrates the fitted factors for NordPool and BBCE, respectively.

PCA NordPool: Fitted Factor Loadings

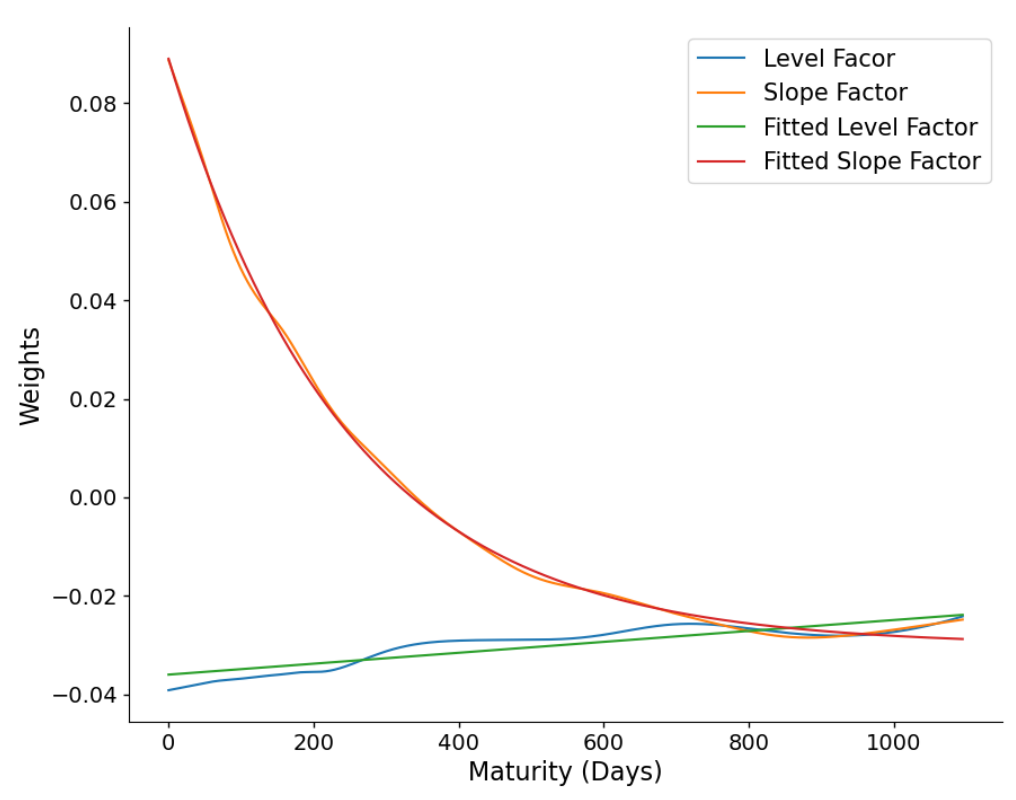

Figure 5.23: Fitted level and slope factors for NordPool. 


\section{PCA BBCE: Fitted Factor Loadings}

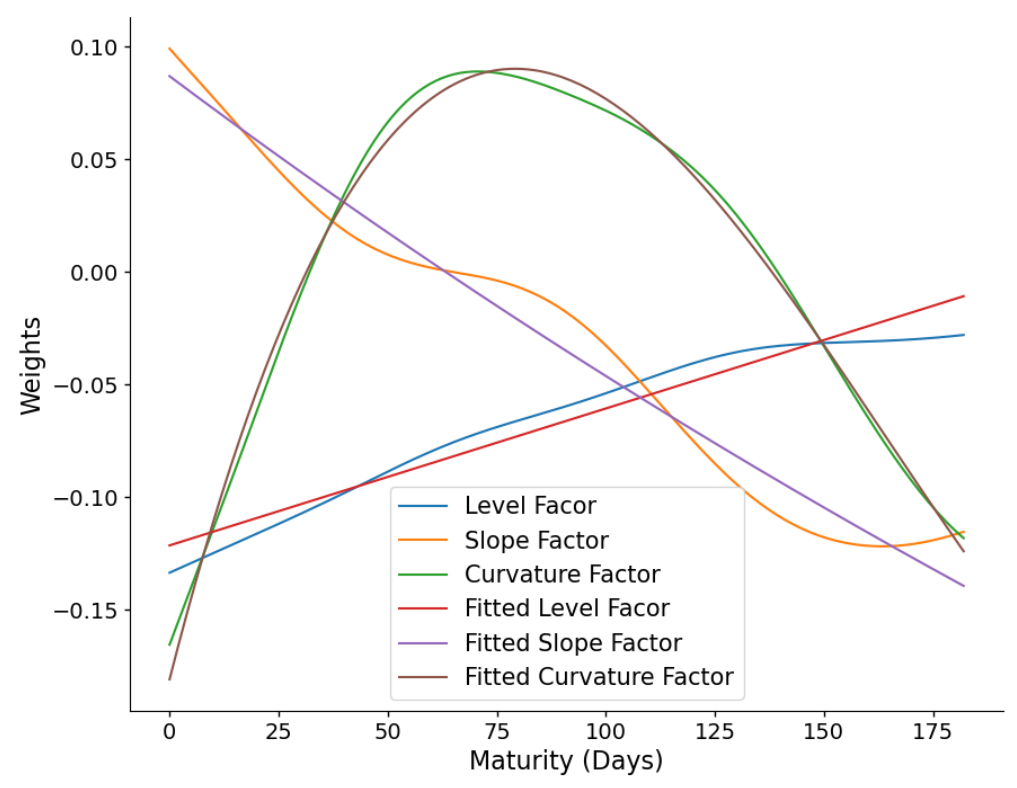

Figure 5.24: Fitted level, slope and curvature factors for BBCE.

To confirm that using low dimensional data weighted by the approximated factor loadings recovers the dynamics of the arbitrage-free swap prices, we calculate the residuals as:

$$
\varepsilon_{t, j}=\mu_{j}+\sum_{k \in \mathcal{K}} w_{j, k} c_{t, k}, \quad \forall t \in \mathcal{T}, \forall j \in \mathcal{J}
$$

Where $w_{j, k}$ is the weight (factor loading) of component $k \in \mathcal{K}$ on the residual of maturity $j$ and $\mu_{j}$ is the mean of $\varepsilon_{t, j}$, for a fixed $j \in \mathcal{J}$. Then, arbitrage-free swap prices are computed by (4-5). Tables 5.1 and 5.2 shows the MAPE values resulted from the comparison between the arbitrage-free swap prices and the ones recovered by the principal components approximation. 
Table 5.1: MAPE NordPool (2 components)

MAPE NordPool

(\%)

\begin{tabular}{cc|cc|cc}
\hline Contracts & MAPE & Contracts & MAPE & Contracts & MAPE \\
\hline $\mathrm{M}+1$ & 3.8 & $\mathrm{Q}+1$ & 2.7 & $\mathrm{Q}+7$ & 2.9 \\
$\mathrm{M}+2$ & 4.0 & $\mathrm{Q}+2$ & 3.1 & $\mathrm{Q}+8$ & 3.1 \\
$\mathrm{M}+3$ & 3.6 & $\mathrm{Q}+3$ & 3.1 & $\mathrm{Y}+1$ & 1.1 \\
$\mathrm{M}+4$ & 3.8 & $\mathrm{Q}+4$ & 3.3 & $\mathrm{Y}+2$ & 1.0 \\
$\mathrm{M}+5$ & 3.7 & $\mathrm{Q}+5$ & 3.0 & $\mathrm{Y}+3$ & 3.9 \\
$\mathrm{M}+6$ & 3.8 & $\mathrm{Q}+6$ & 2.7 & & \\
\hline
\end{tabular}

Table 5.2: MAPE BBCE (3 components)

\begin{tabular}{cc}
\multicolumn{2}{c}{$\begin{array}{c}\text { MAPE BBCE } \\
(\%)\end{array}$} \\
\hline Contracts & MAPE \\
\hline M+1 & 3.3 \\
M+2 & 3.4 \\
M+3 & 3.9 \\
M+4 & 3.7 \\
M+5 & 2.1 \\
M+6 & 6.1 \\
Q+1 & 2.2 \\
Q+2 & 5.8 \\
\hline
\end{tabular}

Next, we illustrate the recovered prices for contracts with different duration from NordPool and BBCE, respectively. 
$\mathrm{M}+3$

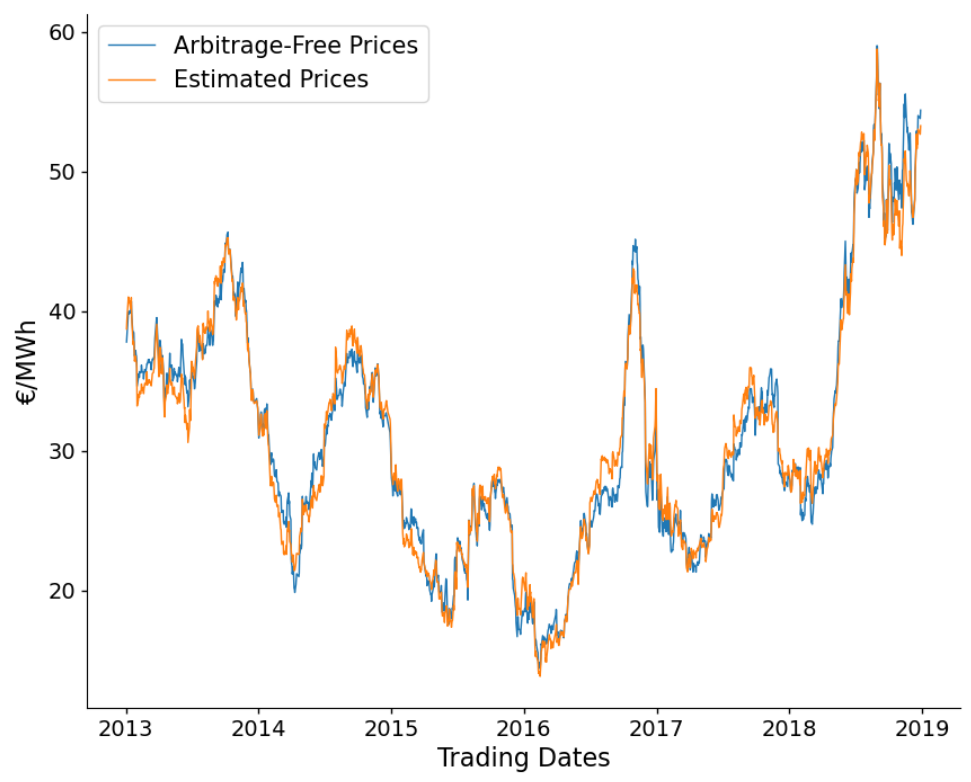

Figure 5.25: $\mathrm{M}+3$ arbitrage-free swaps from NordPool recovered with 2 com-

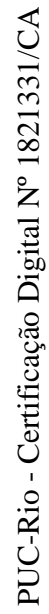
ponents.

$$
\text { Q+1 }
$$

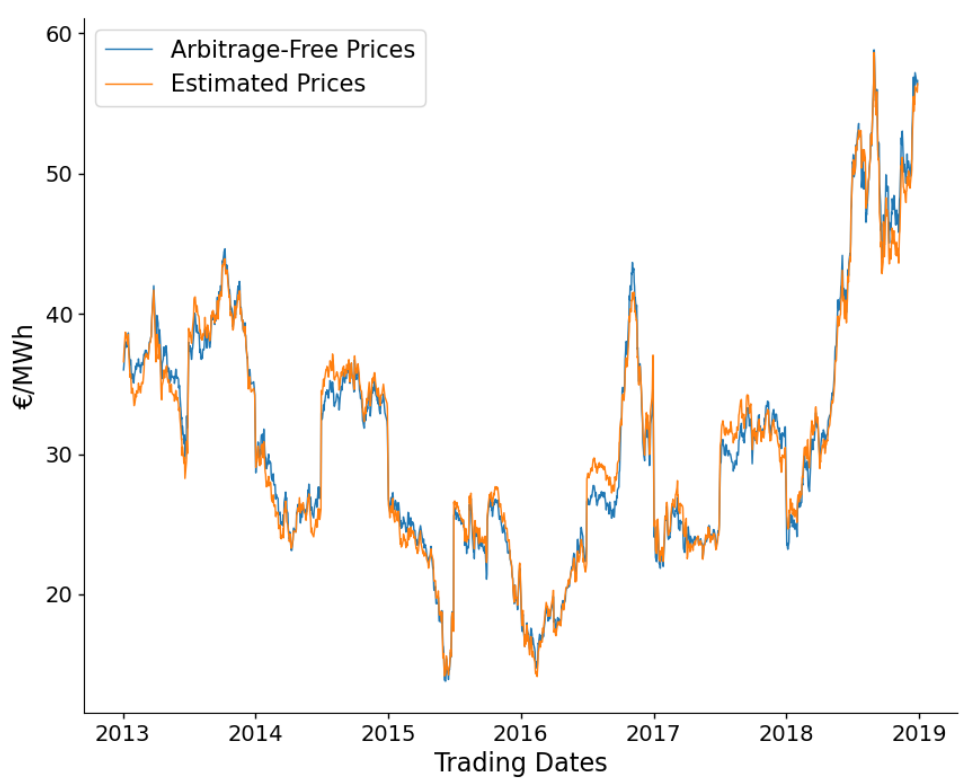

Figure 5.26: Q+1 arbitrage-free swaps from NordPool recovered with 2 components. 


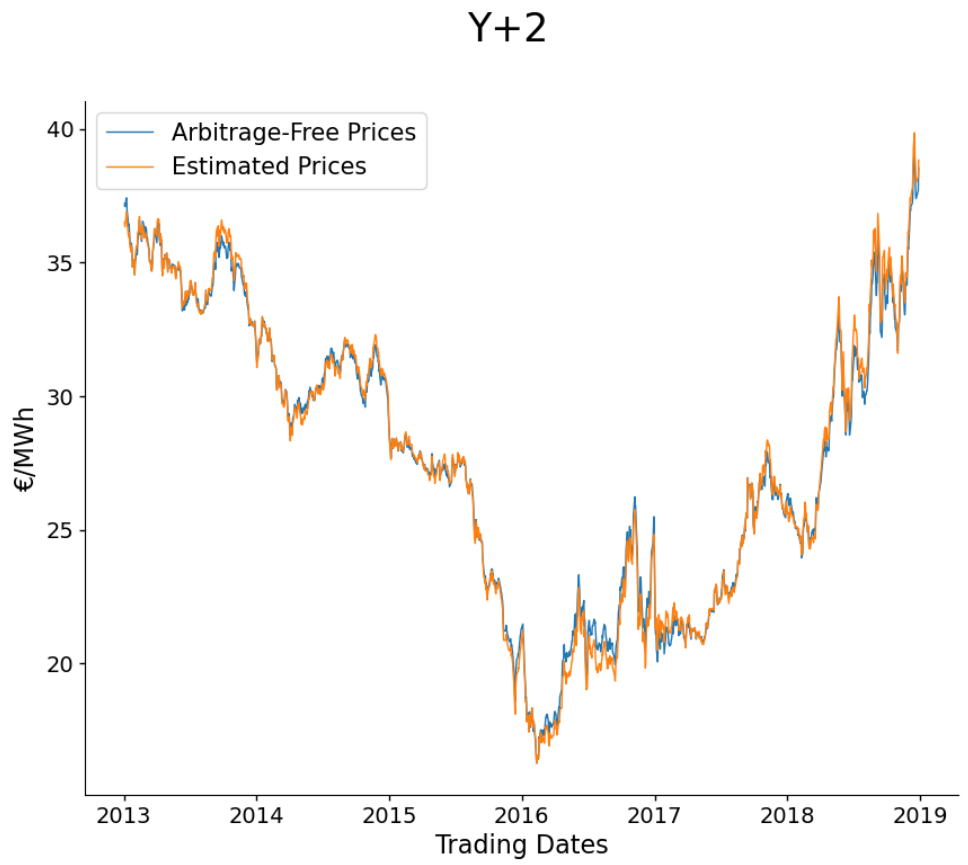

Figure 5.27: Y+2 arbitrage-free swaps from NordPool recovered with 2 components.

$$
M+1
$$

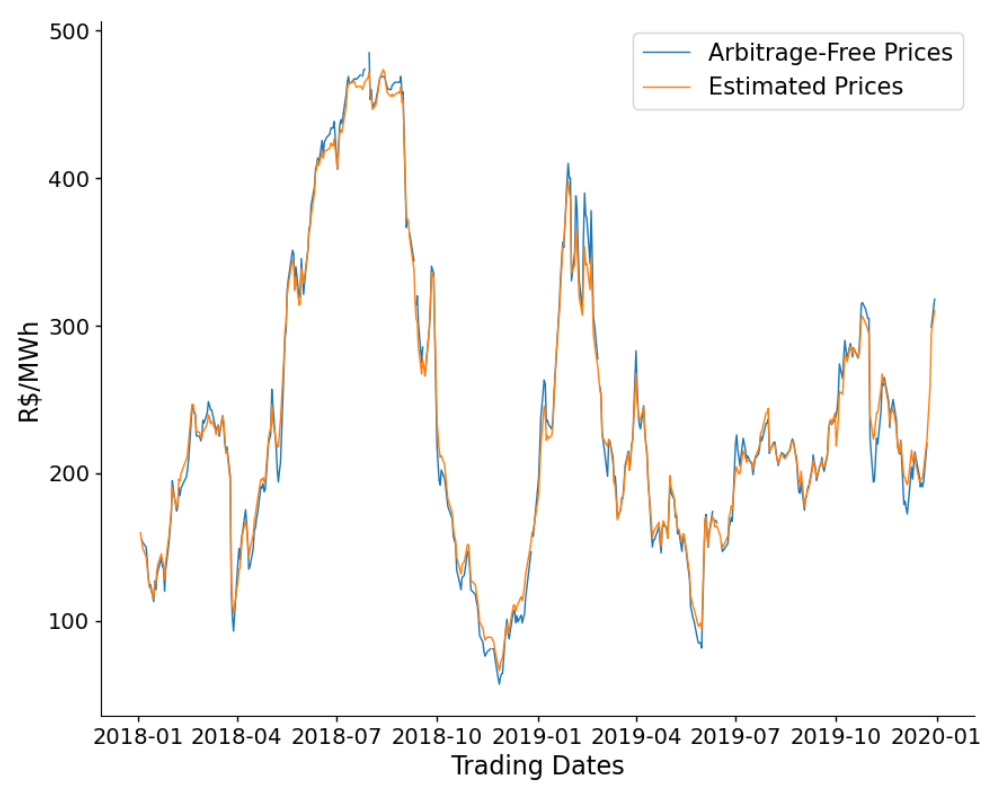

Figure 5.28: $\mathrm{M}+1$ arbitrage-free swaps from BBCE recovered with 3 components. 


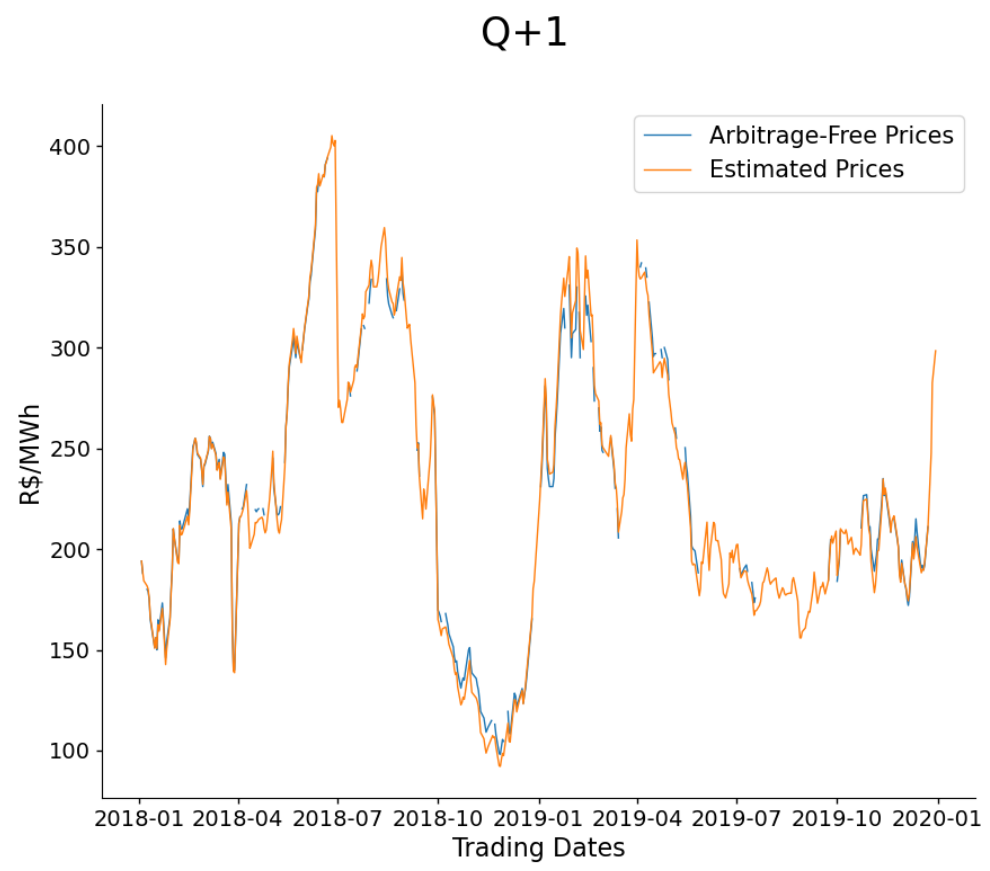

Figure 5.29: $\mathrm{Q}+1$ arbitrage-free swaps from BBCE recovered with 3 components.

The MAPE values found in both markets are coherent with the percentage of the residuals variability explained by the extracted components. For $\mathrm{BBCE}$, the swaps $\mathrm{M}+6$ and $\mathrm{Q}+2$ presented the highest MAPE values. Since there are high levels of missing data, the MAPE is computed only when arbitrage-free prices are available for comparison. Therefore, this might impact the metric values found in the Brazilian market. Nevertheless, the results presented here corroborate with the capacity of the low-dimensional data derived from PCA to recover the arbitrage-free swap prices accurately. 


\section{6 \\ Conclusions}

This study presents a semiparametric structural model to extract continuous forward curves of electricity, composed of elementary forward contracts. The estimation procedure consists of a hierarchical optimization problem where the first stage acknowledges the arbitrage opportunities on power markets due to overlapped contracts and allows some outlier detection. Then, the parameters of the seasonality curve (or any other structure imposed on elementary prices) are estimated through OLS, followed by the computation of the forward curve. In this stage, the maximum smoothness criterion can be applied on the maturity and time dimension to treat scarce data sets from low-liquidity markets. This has shown to be especially relevant in the Brazilian system. Results highlighted the differences between the Brazilian and Nordic power markets in terms of arbitrage levels. The arbitrage identification procedure was also used to detect outliers and to compute arbitrage-free prices. It is important to highlight that our proposed semi-parametric structure for the elementary forward prices allows a more efficient dimensionality reduction. We show that more than $97 \%$ of the residuals variability can be explained by only three principal components for both the Brazilian (BBCE), 98.4\%, and Nordic (NordPool), $97.4 \%$, electricity markets. Based on these results, we show that even under a reduced dimension we can accurately reconstruct all time series. 


\section{7 \\ Next Steps}

This chapter highlights the main next steps regarding the semiparametric structural model developed in this study. The more immediate one would be its use for forecasting purposes. To better understand how this can be done, we define next a general probabilistic forecasting scheme and present some preliminary results.

\section{1}

\section{Probabilistic Forecasting}

If we look back into our reduced-form model in (4-5), it's easy to verify that, to generate probabilistic forecasts of the arbitrage-free swap prices, we must simulate the residuals $\varepsilon_{t, j}$, as all the structure in $\boldsymbol{X}_{t, i} \boldsymbol{\beta}$ is deterministic after the estimation procedure. Here, an observation is made regarding the consideration exogenous variables. Its scenarios would have been calculated outside our framework, by another statistical model, and used as an input. Thus, when looking to equation (4-5), they can be treated in a deterministic manner.

Therefore, instead of performing PCA directly on an optimized set build by the combination of elementary contracts as [2] and [10], we've reduced the dimension of the residuals $\varepsilon_{t, j}$. An advantage of this approach is similar to applying the maximum smoothness criterion on the residuals: the structure imposed on the elementary forward contracts remains static.

Conducting PCA on the residuals reduces the amount of time series to be modeled to obtain probabilistic forecasts for $Y_{t, i}$. If we disregarded this step, scenarios for each maturity $j \in \mathcal{J}$ should be generated. For daily elementary contracts, the number of maturities can be much greater than a hundred, making this task impracticable. Figure 7.1 illustrates our complete probabilistic forecasting scheme. 


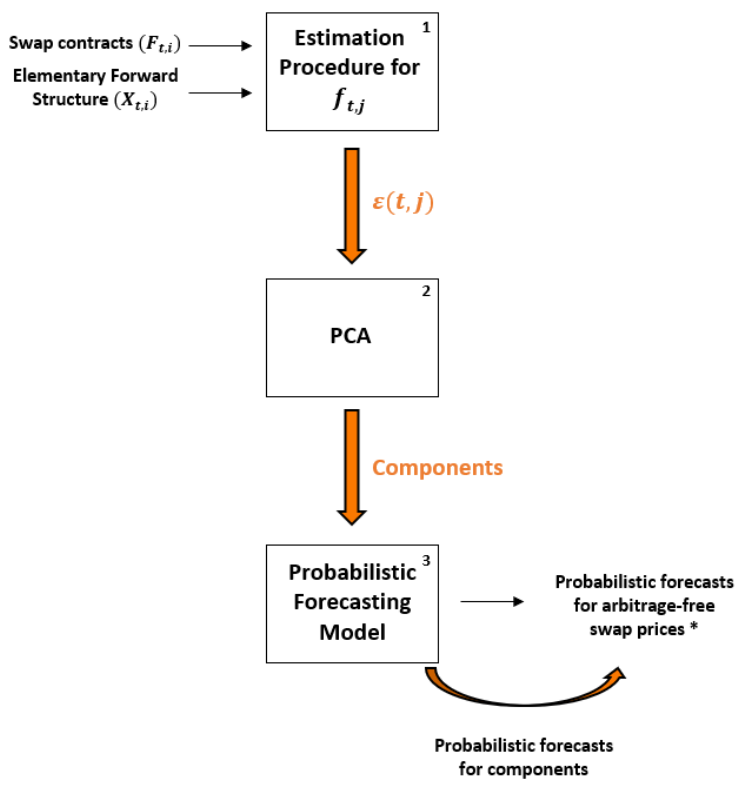

Figure 7.1: Probabilistic forecasting scheme.

The probabilistic forecast scheme's first step is computing the elementary forward contracts and their respective residuals by conducting the estimation procedure developed in Chapter 4. Then, we perform PCA in the data matrix composed by the residuals for each date and maturity and obtain orthogonal time series, called components, and their associated factor loading's (weights). We have already discussed the ability of our framework in explaining high percentages of the total variance of the data with well-behaved factor loadings.

As can be observed in Figure 7.1, the probabilistic forecast model has as input the components resulted from PCA. These time series are uncorrelated, which means univariate models can fit each of them separately. As an initial study in our forecasting procedure, we've estimated an $\operatorname{AR}(1)-\operatorname{GARCH}(1,1)$ for the components of NordPool, which is a discussed structure on the real-time and day-ahead price prediction.

After achieving all the previous stages, the scenarios for the arbitrage-free prices are produced by following the same steps in the opposite order. Figure 7.2 illustrates the procedure with more details. 


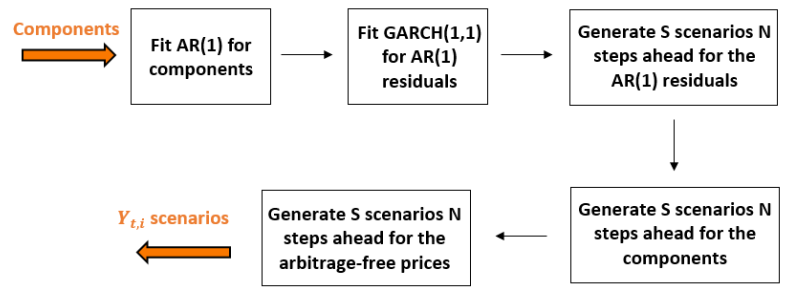

Figure 7.2: Calculation of arbitrage-free prices scenarios.

Let $c_{t, k}$ be the value of the extracted component $k \in \mathcal{K}$ on trading date t. Then:

$$
c_{t, k}=\alpha+\phi c_{t-1, k}+\xi_{t, k}, \quad \forall t \in \mathcal{T}, \forall k \in \mathcal{K}
$$

With $\xi_{t, k} \sim \operatorname{GARCH}(1,1):$

$$
\begin{array}{ll}
\xi_{t, k}=\mu+\sigma_{t, k} z_{t, k}, & \forall t \in \mathcal{T}, \forall k \in \mathcal{K} \\
\sigma_{t, k}^{2}=\omega+\alpha\left(\xi_{t, k}-\mu\right)^{2}+\beta \sigma_{t-1, k}^{2}, & \forall t \in \mathcal{T}, \forall k \in \mathcal{K}
\end{array}
$$

where $z_{t, k} \sim N(0,1)$. After estimating all parameters of (7-1)-(7-3), we can simulate the residuals $\xi_{t, k}$ iteratively through the equations (7-2)-(7-3). In addition, the components scenarios are calculated through (7-1) by replacing $\xi_{t, k}$ with the results of the previous simulation.

From the PCA formulation, we write the residuals $\varepsilon_{t, j}$ as a linear combination of the extracted components, weighted by their factor loadings:

$$
\varepsilon_{t, j}=\mu_{j}+\sum_{k \in \mathcal{K}} w_{j, k} c_{t, k}, \quad \forall t \in \mathcal{T}, \forall j \in \mathcal{J}
$$

Where $w_{j, k}$ is the weight of component $k \in \mathcal{K}$ on the residual of maturity $j$ and $\mu_{j}$ is the mean of $\varepsilon_{t, j}$, for a fixed $j \in \mathcal{J}$. The scenarios for $\varepsilon_{t, j}$ are a result of (7-4) with the simulation of components being replaced in $c_{t, k}$. Finally, scenarios of arbitrage-free prices are computed with (4-5).

To evaluate our probabilistic forecasting model, we've performed 100 iterations of the scheme in Fig. 7.1 on a rolling horizon strategy. In each iteration, we've generated 1000 prices scenarios, up to 20 steps ahead, and computed the probabilistic calibration metric [20], that reflects the consistency between the forecast's distribution and the observations. For example, we 
expect that $95 \%$ of the time, the observed prices are lower than the quantile of $95 \%$, and so forth. It's a property that connects the forecasts and materialized events and translates the prediction model's reliability.

To investigate the prediction model reliability, we show, in Tables 7.1, 7.2 and 7.3 , the percentage of iterations where the observed prices were below the quantiles specified in the first column, for the monthly, quarterly and yearly swaps. Thus, if a probabilistic forecast is perfectly calibrated, the values on each line of all tables would coincide with the correspondent quantiles. 
Table 7.1: Probabilistic calibration for monthly swaps from NordPool

Probabilistic Calibration

(\%)

\begin{tabular}{|c|c|c|c|c|c|c|}
\hline \multicolumn{7}{|c|}{ Monthly Contracts } \\
\hline \multicolumn{7}{|c|}{1 STEP AHEAD } \\
\hline Quantiles & $M+1$ & $M+2$ & $M+3$ & $M+4$ & $M+5$ & $M+6$ \\
\hline 7.5 & 13 & 13 & 12 & 8 & 2 & 8 \\
\hline 17.5 & 16 & 16 & 12 & 9 & 4 & 11 \\
\hline 27.5 & 21 & 20 & 14 & 10 & 4 & 14 \\
\hline 37.5 & 24 & 21 & 18 & 10 & 6 & 17 \\
\hline 47.5 & 26 & 22 & 22 & 12 & 6 & 19 \\
\hline 57.5 & 29 & 23 & 24 & 13 & 7 & 21 \\
\hline 67.5 & 31 & 28 & 26 & 14 & 7 & 22 \\
\hline 77.5 & 33 & 31 & 29 & 15 & 7 & 22 \\
\hline 87.5 & 41 & 37 & 33 & 17 & 9 & 24 \\
\hline 97.5 & 54 & 45 & 39 & 30 & 12 & 30 \\
\hline
\end{tabular}

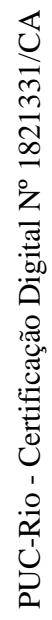

5 STEPS AHEAD

\begin{tabular}{c|cccccc}
\hline Quantiles & $\mathbf{M}+\mathbf{1}$ & $\mathbf{M + 2}$ & $\mathbf{M + 3}$ & $\mathbf{M + 4}$ & $\mathbf{M + 5}$ & $\mathbf{M + 6}$ \\
\hline $\mathbf{7 . 5}$ & 9 & 9 & 6 & 3 & 2 & 5 \\
$\mathbf{1 7 . 5}$ & 15 & 13 & 8 & 5 & 2 & 5 \\
$\mathbf{2 7 . 5}$ & 19 & 15 & 12 & 8 & 3 & 5 \\
$\mathbf{3 7 . 5}$ & 24 & 17 & 13 & 9 & 5 & 6 \\
$\mathbf{4 7 . 5}$ & 26 & 18 & 15 & 11 & 5 & 6 \\
$\mathbf{5 7 . 5}$ & 29 & 22 & 18 & 12 & 7 & 9 \\
$\mathbf{6 7 . 5}$ & 33 & 27 & 23 & 14 & 9 & 14 \\
$\mathbf{7 7 . 5}$ & 38 & 28 & 24 & 18 & 13 & 18 \\
$\mathbf{8 7 . 5}$ & 45 & 36 & 30 & 22 & 15 & 20 \\
$\mathbf{9 7 . 5}$ & 54 & 41 & 34 & 30 & 21 & 29 \\
\hline
\end{tabular}

20 STEPS AHEAD

\begin{tabular}{c|cccccc}
\hline Quantiles & M+1 & M+2 & M+3 & M+4 & M+5 & M+6 \\
\hline $\mathbf{7 . 5}$ & 3 & 0 & 0 & 0 & 0 & 0 \\
$\mathbf{1 7 . 5}$ & 3 & 1 & 2 & 0 & 0 & 0 \\
$\mathbf{2 7 . 5}$ & 5 & 2 & 2 & 0 & 0 & 1 \\
$\mathbf{3 7 . 5}$ & 5 & 2 & 3 & 0 & 0 & 2 \\
$\mathbf{4 7 . 5}$ & 7 & 2 & 4 & 0 & 0 & 2 \\
$\mathbf{5 7 . 5}$ & 7 & 3 & 4 & 1 & 0 & 2 \\
$\mathbf{6 7 . 5}$ & 7 & 4 & 5 & 1 & 0 & 4 \\
$\mathbf{7 7 . 5}$ & 8 & 6 & 6 & 2 & 0 & 4 \\
$\mathbf{8 7 . 5}$ & 11 & 12 & 9 & 3 & 1 & 8 \\
$\mathbf{9 7 . 5}$ & 22 & 23 & 11 & 10 & 6 & 17 \\
\hline
\end{tabular}


Table 7.2: Probabilistic calibration for quarterly swaps from NordPool

Probabilistic Calibration

(\%)

\begin{tabular}{c|cccccccc}
\hline \multicolumn{7}{c}{ Quarterly Contracts } \\
\hline \multicolumn{7}{c}{ 1 STEP } & AHEAD \\
\hline Quantiles & Q+1 & Q+2 & Q+3 & Q+4 & Q+5 & Q+6 & Q+7 & Q+8 \\
\hline $\mathbf{7 . 5}$ & 9 & 3 & 63 & 69 & 43 & 1 & 73 & 76 \\
$\mathbf{1 7 . 5}$ & 11 & 5 & 66 & 75 & 45 & 4 & 83 & 85 \\
$\mathbf{2 7 . 5}$ & 12 & 6 & 69 & 78 & 46 & 4 & 87 & 87 \\
$\mathbf{3 7 . 5}$ & 15 & 8 & 73 & 82 & 47 & 5 & 89 & 92 \\
$\mathbf{4 7 . 5}$ & 15 & 12 & 76 & 85 & 47 & 7 & 91 & 92 \\
$\mathbf{5 7 . 5}$ & 18 & 14 & 77 & 88 & 47 & 8 & 93 & 93 \\
$\mathbf{6 7 . 5}$ & 21 & 14 & 78 & 91 & 50 & 15 & 95 & 96 \\
$\mathbf{7 7 . 5}$ & 22 & 16 & 80 & 91 & 51 & 20 & 97 & 97 \\
$\mathbf{8 7 . 5}$ & 27 & 18 & 84 & 92 & 51 & 31 & 98 & 99 \\
$\mathbf{9 7 . 5}$ & 39 & 28 & 86 & 96 & 56 & 54 & 99 & 100 \\
\hline
\end{tabular}

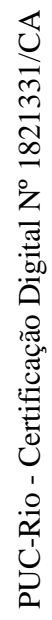

5 STEPS AHEAD

\begin{tabular}{c|cccccccc}
\hline Quantiles & Q+1 & Q+2 & Q+3 & Q+4 & Q+5 & Q+6 & Q+7 & Q+8 \\
\hline $\mathbf{7 . 5}$ & 6 & 1 & 33 & 31 & 11 & 1 & 41 & 34 \\
$\mathbf{1 7 . 5}$ & 10 & 3 & 40 & 37 & 16 & 1 & 53 & 39 \\
$\mathbf{2 7 . 5}$ & 11 & 6 & 47 & 41 & 21 & 2 & 55 & 52 \\
$\mathbf{3 7 . 5}$ & 14 & 6 & 48 & 46 & 26 & 5 & 59 & 62 \\
$\mathbf{4 7 . 5}$ & 18 & 9 & 53 & 50 & 27 & 8 & 67 & 71 \\
$\mathbf{5 7 . 5}$ & 22 & 10 & 54 & 55 & 30 & 10 & 72 & 76 \\
$\mathbf{6 7 . 5}$ & 22 & 12 & 56 & 58 & 31 & 15 & 73 & 79 \\
$\mathbf{7 7 . 5}$ & 26 & 12 & 58 & 67 & 31 & 20 & 77 & 83 \\
$\mathbf{8 7 . 5}$ & 30 & 18 & 67 & 75 & 34 & 30 & 79 & 91 \\
$\mathbf{9 7 . 5}$ & 38 & 36 & 75 & 86 & 53 & 51 & 91 & 97 \\
\hline & & & $\mathbf{2 0}$ & STEPS AHEAD & & & \\
\hline Quantiles & Q+1 & Q+2 & Q+3 & Q+4 & Q+5 & Q+6 & Q+7 & Q+8 \\
\hline $\mathbf{7 . 5}$ & 0 & 0 & 0 & 6 & 0 & 0 & 8 & 2 \\
$\mathbf{1 7 . 5}$ & 2 & 0 & 5 & 7 & 0 & 0 & 18 & 7 \\
$\mathbf{2 7 . 5}$ & 3 & 0 & 14 & 7 & 0 & 0 & 20 & 10 \\
$\mathbf{3 7 . 5}$ & 3 & 0 & 18 & 8 & 0 & 0 & 25 & 11 \\
$\mathbf{4 7 . 5}$ & 4 & 0 & 21 & 11 & 0 & 0 & 29 & 14 \\
$\mathbf{5 7 . 5}$ & 5 & 0 & 29 & 13 & 0 & 0 & 32 & 16 \\
$\mathbf{6 7 . 5}$ & 6 & 1 & 32 & 18 & 0 & 0 & 38 & 17 \\
$\mathbf{7 7 . 5}$ & 7 & 2 & 34 & 21 & 3 & 1 & 43 & 24 \\
$\mathbf{8 7 . 5}$ & 9 & 3 & 39 & 25 & 4 & 2 & 49 & 35 \\
$\mathbf{9 7 . 5}$ & 12 & 22 & 45 & 39 & 11 & 18 & 61 & 60 \\
\hline & & & & & & & &
\end{tabular}


Table 7.3: Probabilistic calibration for yearly swaps from NordPool Probabilistic Calibration

\begin{tabular}{|c|c|c|c|}
\hline \multicolumn{3}{|c|}{ Yearly Contracts } & \\
\hline \multicolumn{4}{|c|}{1 STEP AHEAD } \\
\hline Quantiles & $\mathrm{Y}+1$ & $\mathrm{Y}+2$ & $\mathrm{Y}+3$ \\
\hline 7.5 & 34 & 38 & 8 \\
\hline 17.5 & 42 & 60 & 21 \\
\hline 27.5 & 44 & 69 & 28 \\
\hline 37.5 & 48 & 74 & 30 \\
\hline 47.5 & 54 & 79 & 33 \\
\hline 57.5 & 57 & 82 & 35 \\
\hline 67.5 & 62 & 84 & 41 \\
\hline 77.5 & 65 & 87 & 43 \\
\hline 87.5 & 72 & 89 & 48 \\
\hline 97.5 & 85 & 97 & 61 \\
\hline \multicolumn{4}{|c|}{5 STEPS AHEAD } \\
\hline Quantiles & $\mathrm{Y}+1$ & $\mathrm{Y}+2$ & $Y+3$ \\
\hline 7.5 & 6 & 6 & 4 \\
\hline 17.5 & 7 & 14 & 9 \\
\hline 27.5 & 15 & 24 & 13 \\
\hline 37.5 & 21 & 37 & 19 \\
\hline 47.5 & 24 & 40 & 24 \\
\hline 57.5 & 28 & 46 & 27 \\
\hline 67.5 & 33 & 49 & 33 \\
\hline 77.5 & 37 & 53 & 34 \\
\hline 87.5 & 47 & 60 & 36 \\
\hline 97.5 & 67 & 80 & 53 \\
\hline \multicolumn{4}{|c|}{20 STEPS AHEAD } \\
\hline Quantiles & $Y+1$ & $\mathrm{Y}+2$ & $\mathrm{Y}+3$ \\
\hline 7.5 & 0 & 0 & 0 \\
\hline 17.5 & 0 & 0 & 0 \\
\hline 27.5 & 0 & 0 & 0 \\
\hline 37.5 & 0 & 0 & 1 \\
\hline 47.5 & 1.0 & 0 & 1 \\
\hline 57.5 & 2.0 & 2 & 4 \\
\hline 67.5 & 3.0 & 3 & 5 \\
\hline 77.5 & 3.0 & 7 & 9 \\
\hline 87.5 & 9.1 & 16 & 12 \\
\hline 97.5 & 30.3 & 37 & 39 \\
\hline
\end{tabular}


It is easy to notice that modeling the components with an $\mathrm{AR}(1)$ GARCH $(1,1)$ framework did not produce good probabilistic calibration metrics. Nevertheless, the dynamics of some swap series could be recovered by our forecasting procedure. Fig. 7.3 and 7.4 illustrates the scenarios for the $\mathrm{Q}+7$ and $\mathrm{Y}+2$ time series for a window of the rolling horizon.

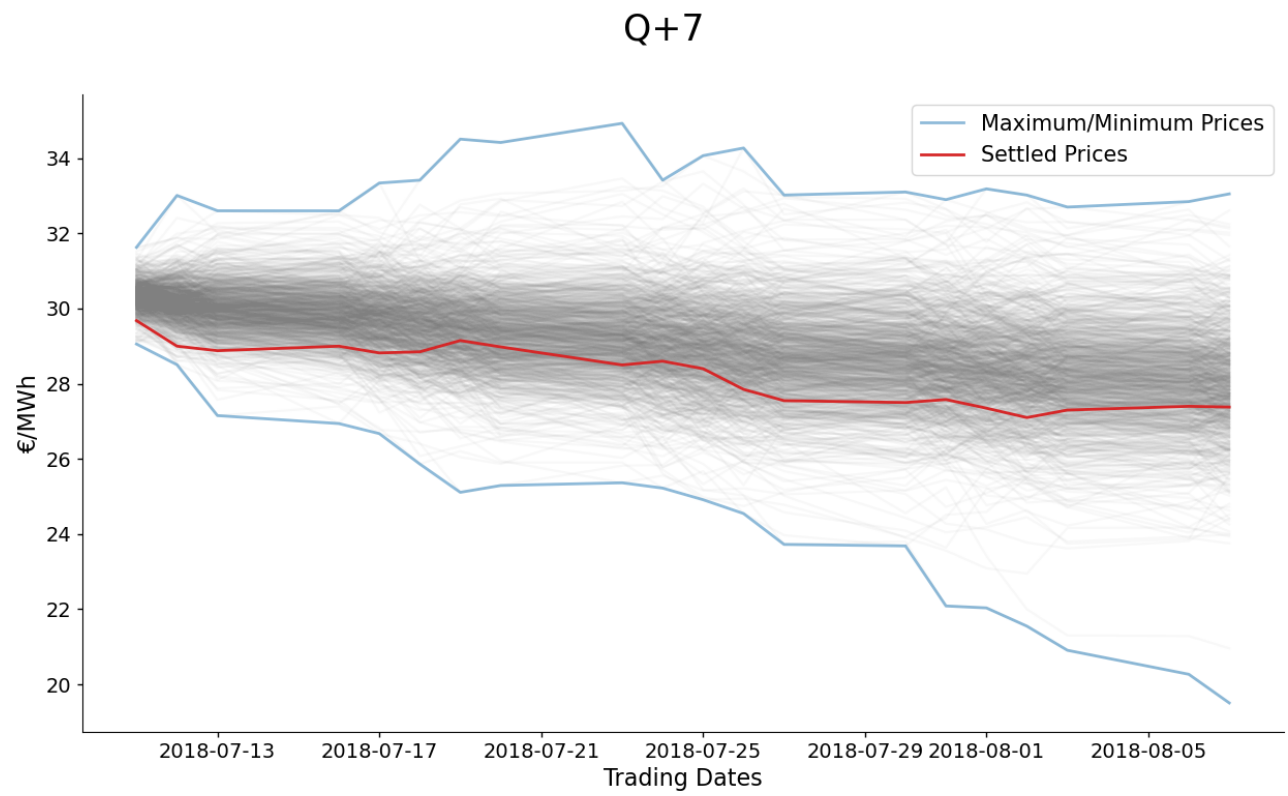

Figure 7.3: Simulation of Q+7 NordPool prices.

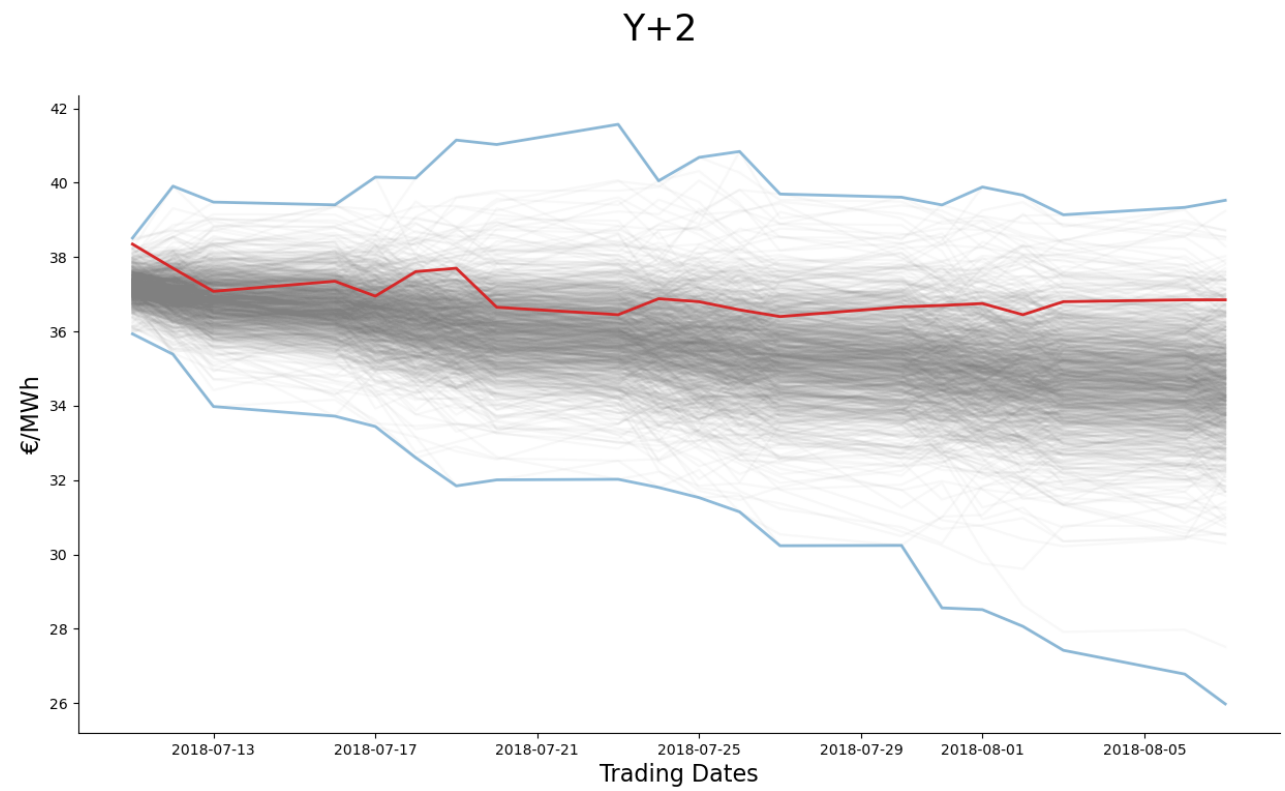

Figure 7.4: Simulation of Y+2 NordPool prices. 
It's important to recognize that perhaps the third component's disposal may have negatively impacted the results found, which means that the factors that explain most of the total variance are not necessarily the most suitable for forecasting.

\section{2}

\section{Considerations}

After observing the discussions and results presented in this document, some subsequent evaluations could leverage the proposed framework's potential.

1. Add explanatory variables into the structural model: Some straightforward variables would be spot prices and/or reservoir and inflow levels, for hydro-dominant power systems. Nevertheless, understanding how those variables impact different maturities would be essential to define their appropriate parametric relation with elementary prices.

2. Define other seasonal patterns to remove the remaining structure in the component on the NordPool data set. Also, it could be interesting to differ weekdays from weekends/holidays.

3. Recognize how each component impacts the distinct parts of the forward curve. A derivation of PCA, called Sparse Principal Component Analysis, could help in this process since it aims to find sparse factors. The interpretability of the factors is even more straightforward since the components that affect each maturity would have non-zero weights.

4. Given a parametric form for the factor loadings, it is possible to formulate an optimization problem that incorporates all the concepts and equations developed in Chapter 2 and the component extraction simultaneously. Thus, the residuals of the elementary forward contracts must be written as a linear combination between components and factor loadings. A constraint guaranteeing orthogonality among components is also needed.

5. Define a metric to evaluate the impact of different values of $\lambda_{1}$ and $\lambda_{2}$ on the estimation process.

6. Test different forecasting models to improve the results presented before in this chapter. A possibility would be the class of Score Driven Models [21], which gives high flexibility when choosing the most suitable distribution and how it evolves through time. 
A

\section{Nomenclature}

This section presents the nomenclature and symbols used in this work.

\section{Sets}

$\mathcal{T} \quad$ Set of trading dates $\mathcal{T}=\left\{t_{0}, t_{1}, \ldots, T\right\}$

$\mathcal{J} \quad$ Set of elementary maturity in days $\mathcal{J}=\{1,2, \ldots, J\}$

$\mathcal{N}_{t} \quad$ Set of negotiated swaps on trading date $t \in \mathcal{T}$

$\mathcal{K} \quad$ Set of components indexes

\section{Constants}

$F_{t, i} \quad$ Price of the swap $i$ on trading date $t$

$h_{t, i} \quad$ Time to maturity of the swap $i$ on trading date $t$

$\Delta T_{t, i} \quad$ Duration of the swap $i$ on trading date $t$

$r \quad$ Discount rate

$w_{j, k} \quad$ Factor loading of component $k$ for maturity $j$

\section{Decision Variables}

$f_{t, j} \quad$ Price of the daily elementary forward contract of maturity $j$ on trading date $t$

$\varepsilon_{t, j} \quad$ Residual of the elementary forward contract of maturity $j$ on trading date $t$

$\Delta_{t, i} \quad$ Net present value of the arbitrage level of the swap $i$ traded on date $t$

$\eta_{t, i} \quad$ Adjustment term 
$\zeta_{t, i} \quad$ Arbitrage level in prices of the swap $i$ traded on date $t$

$\boldsymbol{\beta} \quad$ Vector of coefficients of $\boldsymbol{x}_{t, j}$

$c_{t, k} \quad$ Component $k$ in the trading date $t$

\section{Vectors}

$\boldsymbol{x}_{t, j} \quad$ Vector that defines the structure imposed on the elementary forward contract of maturity $j$ on trading date $t$

$\boldsymbol{\zeta}_{t} \quad$ Vector composed by the arbitrage levels on the trading date $t$

\section{Functions}

$\varepsilon(t, j) \quad$ Representation of the elementary forward contracts residuals as a function of time and maturity 
B

\section{Descriptive Statistics}

This section presents some descriptive statistics of the swap price timeseries from NordPool and BBCE.

Table B.1: Descriptive Statistics from NordPool time-series

\begin{tabular}{c|c|c|c|c|c}
\hline \multicolumn{5}{c}{ NordPool } \\
\hline Series & Missing Data (\%) & Mean & Minimum & Maximum & Std \\
\hline M+1 & 0 & 31.3 & 9.9 & 59.4 & 9.2 \\
M+2 & 0 & 31.2 & 13.7 & 58.85 & 9.1 \\
M+3 & 0 & 31.1 & 14.38 & 59.15 & 9.0 \\
M+4 & 0 & 31.1 & 14.05 & 59 & 8.9 \\
M+5 & 0 & 31.0 & 12 & 61 & 8.6 \\
M+6 & 0 & 30.7 & 13 & 60.05 & 8.3 \\
Q+1 & 0 & 31.1 & 13.82 & 58.7 & 8.8 \\
Q+2 & 0 & 30.6 & 13.3 & 57.45 & 8.0 \\
Q+3 & 0 & 29.6 & 12.63 & 49.5 & 6.8 \\
Q+4 & 0 & 29.1 & 16.63 & 45.55 & 6.4 \\
Q+5 & 0 & 28.7 & 14.3 & 47.2 & 6.8 \\
Q+6 & 0 & 28.3 & 13.1 & 43 & 6.6 \\
Q+7 & 0 & 27.7 & 15.1 & 41.75 & 6.0 \\
Q+8 & 0 & 27.6 & 15.95 & 43.05 & 6.1 \\
Y+1 & 0.4 & 29.2 & 16.3 & 47.5 & 6.2 \\
Y+2 & 0 & 27.6 & 16.25 & 39.43 & 5.4 \\
Y+3 & 0 & 27.1 & 16.15 & 38.25 & 5.1 \\
\hline
\end{tabular}


Table B.2: Descriptive Statistics from BBCE time-series

\begin{tabular}{c|c|c|c|c|c}
\hline \multicolumn{5}{c}{ BBCE } \\
\hline Series & Missing Data (\%) & Mean & Minimum & Maximum & Std \\
\hline M+1 & 1.01 & 238.7 & 57.0 & 485.0 & 103.8 \\
M+2 & 8.06 & 233.9 & 79.0 & 448.0 & 82.8 \\
M+3 & 35.69 & 230.5 & 98.0 & 397.0 & 73.5 \\
M+4 & 61.29 & 219.9 & 111.8 & 340.0 & 60.0 \\
M+5 & 83.67 & 217.6 & 135.0 & 350.0 & 50.7 \\
M+6 & 93.55 & 202.9 & 150.0 & 295.0 & 32.0 \\
Q+1 & 29.44 & 229.5 & 99.3 & 394.0 & 67.0 \\
Q+2 & 53.83 & 209.0 & 142.5 & 358.0 & 53.1 \\
\hline
\end{tabular}




\section{Bibliography}

[1] J. J. Lucia and E. S. Schwartz, "Electricity prices and power derivatives: Evidence from the nordic power exchange," Review of Derivatives Research, vol. 5, no. 1 , pp. 5-50, 2002.

[2] S. Koekebakker and F. Ollmar, "Forward curve dynamic in the nordic electricity market," Managerial Finance, vol. 31, no. 6, pp. 73-94, 2005.

[3] M. Povh and S.-E. Fleten, "Modeling long-term electricity forward prices," IEEE Transactions on Power Systems, vol. 24, no. 4, pp. 1649-1656, 2009.

[4] M. G. Figueroa and Cartea, "Pricing in electricity markets: A mean reverting jump diffusion model with seasonality," Applied Mathematical Finance, vol. 12, no. 4, pp. 313-335, 2005.

[5] S.-J. Deng, "Stochastic models of energy commodity prices and their applications: Mean-reversion with jumps and spikes," 2000.

[6] H. Geman and A. Roncoroni, "Understanding the fine structure of electricity prices," The Journal of Business, vol. 79, no. 3, pp. 1225-1261, 2006.

[7] R. Weron, M. Bierbrauer, and S. Trueck, "Modeling electricity prices: Jump diffusion and regime switching," Physica A: Statistical Mechanics and its Applications, vol. 336, no. 1-2, pp. 39-48, 2004.

[8] R. Weron, I. Simonsen, and P. Wilman, Modeling highly volatile and seasonal markets: evidence from the nord pool electricity market, pp. 182-191.

[9] A. Escribano, J. I. Peña, and P. Villaplana, "Modelling electricity prices: International evidence," Oxford Bulletin of Economics and Statistics, vol. 73, no. 5, pp. 622-650, 2011.

[10] F. E. Benth, J. S. Benth, and S. Koekebakker, Stochastic modelling of electricity and related markets. World Scientific, 2008, vol. 11.

[11] K. J. Adams and D. R. V. Deventer, "Fitting yield curves and forward rate curves with maximum smoothness," Journal of Fixed Income, vol. 4, no. 1, pp. 52-62, 1994. 
[12] S.-E. Fleten and J. Lemming, "Constructing forward price curves in electricity markets," Energy Economics, vol. 25, no. 5, pp. 409-424, 2003.

[13] G. Cortazar and E. S. Schwartz, "The valuation of commodity contingent claims," Journal of Derivatives, vol. 1, no. 4, pp. 27-39, 1994.

[14] F. E. Benth and F. Paraschiv, "A space-time random field model for electricity forward prices," Journal of Banking and Finance, vol. 95, pp. 203-216, 2018.

[15] E. Schwartz and J. E. Smith, "Short-term variations and long-term dynamics in commodity prices," Management Science, vol. 46, no. 7, pp. 893-911, 2000.

[16] R. Weron, "Electricity price forecasting: A review of the state-of-the-art with a look into the future," International Journal of Forecasting, vol. 30, no. 4, pp. 1030-1081, 2014.

[17] D. Heath, R. Jarrow, and A. Morton, "Bond pricing and the term structure of interest rates: A new methodology for contingent claims valuation," Econometrica: Journal of the Econometric Society, pp. 77-105, 1992.

[18] R. Kiesel, F. Paraschiv, and A. Sæther $\varnothing$, "On the construction of hourly price forward curves for electricity prices," Computational Management Science, 2018.

[19] F. E. Benth, S. Koekkebakker, and F. Ollmar, "Extracting and applying smooth forward curves from average-based commodity contracts with seasonal variation," The Journal of Derivatives, vol. 15, no. 1, pp. 52-66, 2007.

[20] T. Gneiting, F. Balabdaoui, and A. E. Raftery, "Probabilistic forecasts, calibration and sharpness," Journal of the Royal Statistical Society: Series $B$, vol. 69, no. 2, pp. 243-268, 2007.

[21] D. Creal, S. J. Koopman, and A. Lucas, "Generalized autoregressive score models with applications," Journal of Applied Econometrics, vol. 28, pp. 777795, 2013. 